\title{
Une généralisation du théorème de Liouville effectif pour les variétés projectives
}

\author{
François Ballaÿ
}

\author{
Beijing International Center for Mathematical Research, Peking University \\ 5 Yi He Yuan Road, Beijing 100871, China. \\ francois.ballay@bicmr.pku.edu.cn
}

\section{Résumé}

On démontre un analogue du théorème de Liouville effectif valable pour des points fermés sur une variété projective définie sur un corps de nombres. Ce résultat est une version effective d'un théorème récent de McKinnon et Roth. Une partie importante de cet article est consacrée à la démonstration d'une version effective d'un cas particulier d'un théorème remarquable de géométrie diophantienne dû à Faltings et Wüstholz. Combiné à de nouvelles comparaisons explicites entre l'évaluation d'une section d'un fibré en droites et une fonction distance donnée, ce résultat entraîne le théorème principal. Nous présentons également une autre approche, en montrant comment rendre effectifs les arguments de McKinnon et Roth. Ces deux points de vue conduisent à des versions distinctes $\mathrm{du}$ théorème principal, fournissant des majorations différentes de la hauteur des points satisfaisant une inégalité analogue à celle du théorème de Liouville classique.

\begin{abstract}
We prove an effective analogue of Liouville's theorem for closed points on an arbitrary projective variety defined over a number field. Our result can be interpreted as an effective version of a recent theorem proved by McKinnon and Roth. A central part of the paper is dedicated to giving an effective proof of a particular case of a powerful theorem in diophantine geometry proved by Faltings and Wüstholz. This result, combined with new explicit comparisons between evaluation of sections of a line bundle and a given distance function, leads to the expected theorem. We also deal with another approach, showing how to make the arguments of McKinnon and Roth effective. These two points of view lead to distinct versions of our main result, giving different upper bounds for the height of points satisfying a Liouville type inequality.
\end{abstract}

2010 Mathematics Subject Classification : Primary 14G05, Secondary 14G40, 11J68.

Mots-clés : Approximation diophantienne, théorème de Liouville, géométrie d'Arakelov, constante de Seshadri, points rationnels.

Keywords : Diophantine approximation, Liouville's Theorem, Arakelov Geometry, Seshadri constant, rational points.

Titre court: Généralisation du théorème de Liouville effectif.

English title : An effective generalization of Liouville's theorem for projective varieties. (Running Head : Effective generalization of Liouville's theorem). 


\section{Introduction}

Le théorème de Liouville (1844) affirme que si $x$ est un nombre réel algébrique de degré $d \geq 2$ sur $\mathbb{Q}$, alors pour tout $\varepsilon>0$ il n'existe qu'un nombre fini de solutions rationnelles $p / q \in \mathbb{Q}$ à l'inégalité

$$
\left|x-\frac{p}{q}\right|<\frac{1}{q^{d+\varepsilon}} .
$$

Le théorème de Roth (voir par exemple [9, D.3]) affirme que ce résultat reste valide en remplaçant l'exposant $d$ par 2 . Il existe également une version effective $\mathrm{du}$ théorème de Liouville (voir [9, D.1.2.2]), qui fournit une constante explicite $c(x)>0$ telle que pour tout $p / q \in \mathbb{Q}$, on ait

$$
\left|x-\frac{p}{q}\right|>\frac{c(x)}{q^{d}} .
$$

En revanche, aucune version effective du théorème de Roth n'existe à l'heure actuelle. Dans cet article, on démontre un analogue de l'inégalité (1) valable pour des points algébriques sur une variété projective définie sur un corps de nombres.

Dans l'article [6], Faltings et Wüstholz démontrent une généralisation du théorème de Roth. Considérons une variété projective $X$ définie sur un corps de nombres $K$ et un fibré inversible très ample $\mathscr{L}$ sur $X$. Notons $\Sigma_{K}$ l'ensemble des places de $K$. Le choix d'une base de $\mathrm{H}^{0}(X, \mathscr{L})$ munit le fibré $\mathscr{L}$ d'une métrique $\left(\|\cdot\|_{v}\right)_{v \in \Sigma_{K}}$, définie à l'exemple 2.4. On note $h_{\mathscr{L}}$ la hauteur logarithmique associée sur les points de $X(\bar{K})$ (voir le paragraphe 2.3 ; si $X=\mathbb{P}_{K}^{n}$ est un espace projectif et $\mathscr{L}=\mathscr{O}(1)$, alors $h_{\mathscr{L}}$ est simplement la hauteur de Weil logarithmique sur $\left.\mathbb{P}^{n}(\bar{K})\right)$. On considère une extension finie $L$ de $K$ et un ensemble fini $\mathscr{P}$ de places de $L$. Pour toute place $w \in \mathscr{P}$, on munit le $L$-espace vectoriel $V=\mathrm{H}^{0}(X, \mathscr{L}) \otimes_{K} L$ d'une $\mathbb{R}$-filtration $\mathscr{F}_{w}$, correspondant à un drapeau

$$
V=V_{0}^{(w)} \supsetneq V_{1}^{(w)} \supsetneq \cdots \supsetneq V_{e_{w}}^{(w)} \supsetneq V_{e_{w}+1}^{(w)}=\{0\}
$$

et à une suite de nombres réels

$$
0 \leq c_{w, 0}<c_{w, 1}<\cdots<c_{w, e_{w}} .
$$

On considère une base $\left(s_{w, j, l}\right)_{l}$ de $V_{j}^{(w)}$ pour chaque place $w \in \mathscr{P}$ et chaque $0 \leq j \leq e_{w}$. On note $\mu_{w}(V)$ les pentes des filtrations, définies par

$$
\mu_{w}(V)=\frac{1}{\operatorname{dim} V} \sum_{i=0}^{e_{w}} c_{w, i} \operatorname{dim}\left(V_{i}^{(w)} / V_{i+1}^{(w)}\right) .
$$

Pour tout sous-espace vectoriel non nul $V^{\prime}$ de $V$, on dispose de filtrations induites sur $V^{\prime}$ et on définit $\mu_{w}\left(V^{\prime}\right)$ de la même façon pour chaque place $w \in \mathscr{P}$ (remarquons que $\mu_{w}\left(V^{\prime}\right) \leq c_{w, e_{w}}$ ). On note également

$$
\mu_{\mathscr{P}}\left(V^{\prime}\right)=\sum_{w \in \mathscr{P}}\left[L_{w}: \mathbb{Q}_{w}\right] \mu_{w}\left(V^{\prime}\right) .
$$

On note $W$ le premier saut de la filtration de Harder-Narasimhan associée à la fonction $\mu_{\mathscr{P}}$ au sens de [6, partie 4]. C'est l'unique sous-espace vectoriel de $V$ 
de dimension maximale vérifiant $\mu_{\mathscr{P}}(W)=\max \left\{\mu_{\mathscr{P}}\left(V^{\prime}\right) \mid 0 \neq V^{\prime} \subseteq V\right\}$. On l'appelle le sous-espace déstabilisant associé à $\left(\mathscr{F}_{w}\right)_{w \in \mathscr{P}}$.

Le théorème 9.1 de [6] s'énonce alors de la façon suivante.

Théorème 1.1 (Faltings et Wüstholz, 1994). Supposons que $\mu_{\mathscr{P}}(W)>[L: \mathbb{Q}]$. $I l$ existe une sous-variété stricte $Z$ de $X$ telle que le nombre de points $x \in$ $(X \backslash Z)(K)$ vérifiant

$$
\left\|s_{w, j, l}(x)\right\|_{w} \leq \exp \left(-c_{w, j} h_{\mathscr{L}}(x)\right) \quad \forall w \in \mathscr{P}, \forall j, l
$$

soit fini.

La sous-variété $Z$ du théorème 1.1 est définie comme le lieu de base $\mathscr{B}(W)$ du sous-espace déstabilisant $W$. Le théorème 9.1 de [6] donne donc une définition théorique explicite pour $Z$, bien que cette sous-variété soit très difficile à exhiber en pratique. Dans le cas particulier où les filtrations $\left(\mathscr{F}_{w}\right)_{w \in \mathscr{P}}$ sont conjointement semi-stables (au sens de [6, partie 4]), on a toutefois $W=V$ et $Z=\emptyset$.

Le théorème 1.1 implique le théorème de Roth de la façon suivante. Posons $K=\mathbb{Q}, X=\mathbb{P}_{\mathbb{Q}}^{1}, \mathscr{L}=\mathscr{O}(1)$. Considérons un nombre algébrique $\alpha \in \mathbb{R} \backslash \mathbb{Q}$ et $L$ une extension galoisienne de $\mathbb{Q}$ contenant $\alpha$. Notons $\Sigma_{L, \infty}$ l'ensemble des places infinies de $L$ et fixons $w_{0} \in \Sigma_{L, \infty}$. Pour toute place $w \in \Sigma_{L, \infty}$, il existe un élément $\gamma_{w}$ du groupe de $\operatorname{Galois} \operatorname{Gal}(L / \mathbb{Q})$ tel que $\left|\gamma_{w}^{-1}(x)\right|_{w_{0}}=|x|_{w}$ pour tout $x \in L$. On pose $s_{w}=T_{0}-\gamma_{w}(\alpha) T_{1}$. On munit le fibré inversible $\mathscr{L}=\mathscr{O}(1)$ de la métrique de l'exemple 2.4, qui vérifie : pour tout $x=(p: q) \in \mathbb{P}^{1}(\mathbb{Q})$ avec $p, q$ premiers entre eux,

$$
\left\|s_{w_{0}}(x)\right\|_{w_{0}}=\frac{|p-q \alpha|_{w_{0}}}{\max (|p|,|q|)} \text { et } \quad \exp h_{\mathscr{L}}((p: q))=\max (|p|,|q|) .
$$

Pour tout $x \in \mathbb{P}^{1}(\mathbb{Q})$, on a par ailleurs

$$
\left\|s_{w}(x)\right\|_{w}=\left\|\gamma_{w}\left(s_{w_{0}}(x)\right)\right\|_{w}=\left\|s_{w_{0}}(x)\right\|_{w_{0}} .
$$

Posons $\mathscr{P}=\Sigma_{L, \infty}$. Pour chaque place $w \in \mathscr{P}$, on considère la filtration du $L$ espace vectoriel $V=\mathrm{H}^{0}\left(\mathbb{P}_{L}^{1}, \mathscr{O}(1)\right)$ donnée par le drapeau $V=V_{0}^{(w)} \supsetneq V_{1}^{(w)}=$ $\operatorname{Vect}_{L}\left(s_{w}\right) \supsetneq\{0\}$ et la suite de nombres réels $c_{w, 0}=0<c_{w, 1}=2+\varepsilon$, où $\varepsilon$ est un nombre réel strictement positif. Dans ce cas $\mu_{w}(V)=1+\varepsilon / 2$ pour toute place $w \in \mathscr{P}$ et on peut vérifier que ces filtrations sont conjointement semi-stables. On en conclut qu'il n'existe qu'un nombre fini de points $p / q \in \mathbb{Q}$ tels que

$$
\left|\alpha-\frac{p}{q}\right|_{w_{0}} \leq \frac{1}{|q| \max (|p|,|q|)^{1+\varepsilon}}
$$

et on en déduit le théorème de Roth en remarquant que $\max (|p|,|q|)=O(|q|)$ pour $p / q$ proche de $\alpha$.

McKinnon et Roth démontrent des généralisations du théorème de Roth et du théorème de Liouville sur une variété projective de dimension quelconque dans leurs articles [13] et [14] respectivement. L'article [13] utilise le théorème de Faltings et Wüstholz, tandis que la démonstration de l'analogue du théorème de Liouville repose sur des méthodes différentes (que nous rappellerons dans la partie 3). Soit $X$ une variété projective définie sur un corps de nombres $K, x$ 
un point fermé algébrique de $X$ et $\mathscr{L}$ un fibré inversible nef sur $X$ auquel on associe une fonction de hauteur $h_{\mathscr{L}}$ sur les points de $X(\bar{K})$. On note $L$ le corps de définition de $x$. On fixe une place $v$ de $L$ et une fonction distance $d_{v}$ sur $X$ donnée par un plongement $\varphi: X \hookrightarrow \mathbb{P}_{K}^{n}$ (voir le paragraphe 2.2). On note $\pi: \widetilde{X} \rightarrow$ $X \times_{K} \operatorname{Spec}(L)$ l'éclatement en $x$ et $E$ le diviseur exceptionnel. Le théorème 3.3 de [14] donne la généralisation suivante du théorème de Liouville, dans laquelle intervient le lieu de base stable d'un fibré inversible (nos normalisations de la hauteur et de la distance diffèrent de celles de [14]).

Théorème 1.2 (McKinnon et Roth, 2016). Soit $\gamma>0$ un nombre rationnel tel que $\mathscr{L}_{\gamma}:=\pi^{*} \mathscr{L}-\gamma E$ soit dans le cône effectif de $\operatorname{Pic}_{\mathbb{Q}}(\widetilde{X})$. On note $\mathbf{B}\left(\mathscr{L}_{\gamma}\right)$ le lieu de base stable de $\mathscr{L}_{\gamma}$ et $\mathscr{B}=\pi\left(\mathbf{B}\left(\mathscr{L}_{\gamma}\right)\right)$. Il existe une constante $M \in \mathbb{R}$ telle que, pour tout point $y \in(X \backslash \mathscr{B})(K)$ distinct de $x$, on ait

$$
\log \left(d_{v}(x, y)\right) \geq-\left(\frac{[L: \mathbb{Q}]}{\left[L_{v}: \mathbb{Q} v\right] \gamma}\right) h_{\mathscr{L}}(y)+M .
$$

Dans l'article [14], la constante $M$ qui apparait dans ce théorème n'est pas effective. Dans la partie 3, nous verrons comment adapter les méthodes de [14] afin de l'expliciter, pour obtenir une version effective du théorème 1.2 (théorème 3.1). Cette constante dépend de $X, \varphi, L, \mathscr{L}, x$ et $\gamma$, mais également d'un choix de métriques sur le fibré inversible $\mathscr{O}_{\widetilde{X}}(E)$ associé au diviseur exceptionnel $E$ sur l'éclatement $\widetilde{X}$. Dans un second temps, nous montrerons comment obtenir des bornes qui ne dépendent pas d'un tel choix quand le point $x$ est régulier. Cette seconde approche repose sur des méthodes différentes, inspirées de [13].

Nous commencerons par démontrer des variantes effectives du théorème 1.1 de Faltings et Wüstholz. Soit $\mathscr{L}$ un fibré inversible nef sur $X$ et $\left(\|\cdot\|_{v}\right)_{v \in \Sigma_{K}}$ une métrique adélique sur $\mathscr{L}$ (au sens de la définition 2.3). Nous obtiendrons par exemple le théorème suivant (théorème 4.1).

Théorème 1.3. Soit $F$ un sous-espace vectoriel non nul de $\mathrm{H}^{0}(X, \mathscr{L}) \otimes_{K} L$ et soit $\left(s_{1}, \ldots, s_{\operatorname{dim}_{F}}\right)$ une base de $F$. Soit $\mathscr{P} \subset \Sigma_{L}$ un ensemble fini et soit $\left(t_{w}\right)_{w \in \mathscr{P}}$ une famille de nombres réels telle que

$$
\delta:=\sum_{w \in \mathscr{P}} \frac{\left[L_{w}: \mathbb{Q} w\right]}{[L: \mathbb{Q}]} t_{w}>1 .
$$

Alors tous les points $y \in X(K)$ en dehors du lieu de base de $F$ satisfaisant le système d'inégalités

$$
\frac{\left\|s_{j}(y)\right\|_{w}}{\left\|s_{j}\right\|_{w, \text { sup }}} \leq \exp \left(-t_{w} h_{\mathscr{L}}(y)\right), \quad \forall 1 \leq j \leq \operatorname{dim} F, \quad \forall w \in \mathscr{P}
$$

vérifient

$$
h_{\mathscr{L}}(y) \leq \frac{1}{\delta-1} \max _{1 \leq j \leq \operatorname{dim} F} h_{\mathscr{L}}\left(s_{j}\right) .
$$

En particulier, ces points sont en nombre fini si $\mathscr{L}$ est ample.

La démonstration du théorème 1.3 repose sur des comparaisons élémentaires entre les normes de sections globales de $\mathscr{L}$ et la hauteur des points rationnels de $X$ vérifiant (3), rendues possibles par le formalisme adélique que nous utilisons. Nous présenterons plusieurs dérivés de ce théorème dans la partie 4 (voir 
notamment le corollaire 4.4 pour une formulation analogue à celle du théorème 1.1). Dans le cas particulier où $\cap_{w \in \mathscr{P}} V_{w, e_{w}} \neq\{0\}$ (par exemple lorsque $\operatorname{card}(\mathscr{P})=1$ ), notre approche donne une preuve rapide du théorème 1.1 (en prenant $Z \subsetneq X$ égale au lieu de base de $\left.\cap_{w \in \mathscr{P}} V_{w, e_{w}}\right)$. En général, la condition $\cap_{w \in \mathscr{P}} V_{w, e_{w}} \neq\{0\}$ n'est pas vérifiée avec les hypothèses du théorème 1.1 ; pour cette raison, nos résultats du paragraphe 4 ne permettent pas de retrouver le théorème de Roth. Le principal avantage de nos résultats est qu'ils sont effectifs : ils fournissent une majoration explicite de la hauteur des solutions du système d'inégalités considéré. Cette nouveauté sera le point clé qui nous permettra de démontrer une nouvelle généralisation effective du théorème de Liouville dans la partie 5 . Le lieu de base $\mathscr{B}(F)$ du théorème 1.3 est également explicite, et la condition $y \notin \mathscr{B}(F)$ est facile à vérifier en pratique. En outre, le théorème 1.3 est valable pour un fibré inversible nef (non nécessairement très ample) muni d'une métrique adélique quelconque.

Reprenons les notations introduites avant le théorème 1.2. Concrètement, pour tout nombre rationnel $\gamma>0$ nous donnerons une constante explicite $a(\mathscr{L}, x, \gamma)$ et nous démontrerons le théorème suivant (théorème 5.3 , corollaire 5.5 et remarque 5.6$)$ :

Théorème 1.4. Supposons que le point $x$ est régulier. Soit $\gamma>0$ un nombre rationnel tel que $\mathscr{L}_{\gamma}:=\pi^{*} \mathscr{L}-\gamma E$ soit dans le cône effectif de $\operatorname{Pic}_{\mathbb{Q}}(\widetilde{X})$. On note $\mathbf{B}\left(\mathscr{L}_{\gamma}\right)$ le lieu de base stable de $\mathscr{L}_{\gamma}$ et $\mathscr{B}=\pi\left(\mathbf{B}\left(\mathscr{L}_{\gamma}\right)\right)$. Alors pour tout $\varepsilon>0$, tous les points $y \in(X \backslash \mathscr{B})(K)$ distincts de $x$ satisfaisant l'inégalité

$$
\log \left(d_{v}(x, y)\right)<-\left(\frac{[L: \mathbb{Q}]}{\left[L_{v}: \mathbb{Q}_{v}\right] \gamma}+\varepsilon\right) h_{\mathscr{L}}(y)
$$

vérifient

$$
h_{\mathscr{L}}(y)<\frac{1}{\varepsilon} a(\mathscr{L}, x, \gamma)
$$

Afin d'appliquer le théorème 1.3, nous devrons comparer les normes de sections de $\mathscr{L}$ et la fonction distance. Pour cela, nous démontrons un analogue du lemme de Schwarz pour les sections globales d'un fibré inversible adélique (lemme 5.2). Plus précisément, si $s \in \mathrm{H}^{0}(X, \mathscr{L}) \backslash\{0\}$ s'annule à l'ordre $r \in \mathbb{N} \backslash\{0\}$ en $x$, on montre que pour tout point $y \in X\left(\mathbb{C}_{v}\right)$ tel que $d_{v}(x, y)<1 / 2$, on a

$$
\frac{\|s(y)\|_{v}}{\|s\|_{v, \text { sup }}} \leq\left(\frac{2 d_{v}(x, y)}{\rho_{x}(\mathscr{L}, \varphi)}\right)^{r}
$$

(on obtient un résultat plus précis dans la cas $v \nmid \infty$, voir le lemme 5.2). La quantité $\rho_{x}(\mathscr{L}, \varphi)$, introduite au paragraphe 5 , contrôle la taille d'un voisinage analytique de $x$ dans $X\left(\mathbb{C}_{v}\right)$ sur lequel on peut utiliser un lemme de Schwarz en plusieurs variables usuel. Nous obtenons la majoration (4) en choisissant soigneusement une trivialisation de $\mathscr{L}$ autour de $x$ grâce à des arguments d'interpolation. La constante $a(\mathscr{L}, x, \gamma)$ du théorème 1.4 dépend de $L$ et fait intervenir la quantité $\rho_{x}(\mathscr{L}, \varphi)$. Elle est indépendante d'un choix de métrique sur $\mathscr{O}_{\widetilde{X}}(E)$.

Notons $\epsilon_{x}(\mathscr{L})$ la constante de Seshadri en $x$ (définie à la partie 3 ). Le théorème 1.4 permet de fournir une constante explicite $b(x, \mathscr{L})$ dans le corollaire suivant. Ce résultat peut s'interpréter comme une version effective du corollaire 3.6 de [14] dans le sens où il donne une majoration explicite de la hauteur des solutions. 
Corollaire 1.5. Si $\mathscr{L}$ est ample, alors pour tout $\varepsilon>0$, tous les points $y \in X(K)$ distincts de $x$ satisfaisant l'inégalité

$$
\log \left(d_{v}(x, y)\right)<-\left(\frac{[L: \mathbb{Q}]}{\left[L_{v}: \mathbb{Q}_{v}\right] \epsilon_{x}(\mathscr{L})}+\varepsilon\right) h_{\mathscr{L}}(y)
$$

vérifient

$$
h_{\mathscr{L}}(y) \leq \frac{1}{\varepsilon} b(\mathscr{L}, x)
$$

En particulier ces points sont en nombre fini.

Enfin, nous généraliserons l'inégalité (1) au cas de l'espace projectif $\mathbb{P}_{K}^{n}$ muni du fibré $\mathscr{O}(1)$ (paragraphe 5.2). Dans le cas où $n \geq 2$ et $K \neq L$, nous verrons qu'il est possible d'améliorer l'exposant $\frac{[L: \mathbb{Q}]}{\left[L_{v}: \mathbb{Q}_{v}\right]}$.

La partie 2 regroupe les définitions de distance sur une variété projective et de métrique sur un fibré inversible, et inclut quelques résultats sur le minimum absolu d'un fibré adélique. Dans la partie 3, nous montrons comment adapter les arguments de [14] pour expliciter la constante $M$ du théorème 1.2. La partie 4 est consacrée à la démonstration de variantes effectives du théorème de Faltings et Wüstholz. Nous utiliserons ces résultats dans le cas d'une place $(\mathscr{P}=\{v\})$ dans la dernière partie pour démontrer le théorème 1.4 , en obtenant une constante indépendante d'un choix de métriques sur $\mathscr{O}_{\widetilde{X}}(E)$. Le cas particulier de l'espace projectif $\mathbb{P}_{K}^{n}$ est traité dans le paragraphe 5.2 .

Signalons que dans un travail récent [7], Carlo Gasbarri étudie les points transcendants d'une variété projective définie sur un corps de nombres vérifiant une inégalité similaire à l'inégalité de Liouville. Si le problème étudié ici est différent, un outil commun avec [7] est la comparaison récurrente des normes de sections d'un fibré inversible et de la hauteur de points d'une variété projective. Cet outil, qui découle facilement des définitions, est très classique en approximation diophantienne et il est par exemple au coeur de la démonstration de l'inégalité de Liouville classique ([9, D.3.3]).

Remerciements : Je souhaite exprimer toute ma gratitude à mes deux directeurs de thèse, Huayi Chen et Éric Gaudron, pour leur soutien, leurs conseils et leurs encouragements. Je suis très reconnaissant envers Huayi Chen pour m'avoir suggéré ce problème de recherche, ainsi que pour son suivi précieux. Je remercie également le Laboratoire de Mathématiques Blaise Pascal de l'Université Clermont Auvergne et le Beijing International Center for Mathematical Research de Peking University pour les excellentes conditions de travail qu'ils m'ont offertes.

\section{Table des matières}

1 Introduction

2 Préliminaires

3 L'approche de McKinnon et Roth

4 Autour du théorème de Faltings et Wüstholz 


\section{Préliminaires}

Soit $K$ un corps de nombres.

\subsection{Valeurs absolues et hauteur d'un point projectif.}

On note $\Sigma_{K, f}$ l'ensemble des places finies de $K, \Sigma_{K, \infty}$ l'ensemble de ses places infinies, et $\Sigma_{K}=\Sigma_{K, f} \cup \Sigma_{K, \infty}$. Si $v \in \Sigma_{K}$, on note $K_{v}$ le complété de $K$ en $v$ et $\mathbb{C}_{v}$ le complété d'une clôture algébrique de $K_{v}$. Pour toute place $v \in \Sigma_{K}$, on note $|.|_{v}$ la valeur absolue sur $K$ étendant la valeur absolue $|.|_{v}$ sur $\mathbb{Q}:|p|_{v}=p^{-1}$ si $v$ est une place finie au dessus de $p$ et la restriction de $|\cdot|_{v}$ à $\mathbb{Q}$ est la valeur absolue usuelle si $v$ est archimédienne. On désigne par $\mathbb{Q}_{v}$ le corps $\mathbb{Q}_{p}, \mathbb{R}$ ou $\mathbb{C}$ selon le caractère $p$-adique, réel ou complexe non réel de $v$. On peut alors énoncer la formule du produit : pour tout $x \in K \backslash\{0\}$,

$$
\prod_{v \in \Sigma_{K}}|x|_{v}^{\left[K_{v}: \mathbb{Q}_{v}\right]}=1
$$

Cette formule permet de définir la notion de hauteur d'un point projectif : si $x=\left(x_{0}: \ldots: x_{n}\right) \in \mathbb{P}_{K}^{n}(\bar{K})$ est un point fermé, dont les coordonnées appartiennent à une extension finie $L$ de $K$, on définit sa hauteur (absolue) par

$$
h(x)=\sum_{v \in \Sigma_{L}} \frac{\left[L_{v}: \mathbb{Q}_{v}\right]}{[L: \mathbb{Q}]} \log \left(\max \left\{\left|x_{0}\right|_{v}, \ldots,\left|x_{n}\right|_{v}\right\}\right),
$$

où $\left(x_{0}, \ldots, x_{n}\right)$ est un représentant quelconque de $x$. La hauteur d'un point fermé projectif ne dépend pas du choix du corps $L$. On dispose ainsi d'une notion de hauteur pour tout point $x \in \mathbb{P}^{n}(\bar{K})$. On rencontre fréquemment la définition alternative suivante pour la hauteur d'un point projectif. Pour tout vecteur $\left(z_{0}, \ldots, z_{n}\right) \in \mathbb{C}_{v}^{n+1}$, on pose

$$
\left|\left(z_{0}, \ldots, z_{n}\right)\right|_{2, v}:= \begin{cases}\left(\sum_{i=0}^{n}\left|z_{i}\right|_{v}^{2}\right)^{1 / 2} & \text { si } v \text { est archimédienne } \\ \max \left\{\left|z_{0}\right|_{v}, \ldots,\left|z_{n}\right|_{v}\right\} & \text { sinon. }\end{cases}
$$

Pour tout point $x=\left(x_{0}: \ldots: x_{n}\right) \in \mathbb{P}_{K}^{n}(L)$, on pose

$$
h_{2}(x)=\sum_{v \in \Sigma_{L}} \frac{\left[L_{v}: \mathbb{Q}_{v}\right]}{[L: \mathbb{Q}]} \log \left|\left(x_{0}, \ldots, x_{n}\right)\right|_{2, v} .
$$

De la même façon, $h_{2}(x)$ ne dépend ni du choix de $L$ ni de celui de $\left(x_{0}, \ldots, x_{n}\right)$. Nous définirons une notion de hauteur plus générale associée à un fibré adélique au paragraphe 2.3 .

\subsection{Distance sur une variété projective.}

On considère ici une variété projective $X$ définie $\operatorname{sur} \operatorname{Spec}(K)$ et un plongement $X \hookrightarrow \mathbb{P}_{K}^{n}$. On fixe une place $v_{0}$ de $K$ et une extension de $v_{0}$ à $\bar{K}$, notée $v$. Si $v_{0}$ 
est archimédienne, on définit une fonction distance sur $X(\mathbb{C}) \times X(\mathbb{C})$ en tirant en arrière la fonction sur $\mathbb{P}^{n}(\mathbb{C}) \times \mathbb{P}^{n}(\mathbb{C})$ donnée par

$$
d_{v}(x, y)=\left(1-\frac{\left|\sum_{i=0}^{n} x_{i} \bar{y}_{i}\right|_{v}^{2}}{\left(\sum_{i=0}^{n}\left|x_{i}\right|_{v}^{2}\right)\left(\sum_{i=0}^{n}\left|y_{i}\right|_{v}^{2}\right)}\right)^{1 / 2} .
$$

Si $v_{0}$ est une place finie, on définit une fonction distance sur $X\left(\mathbb{C}_{v}\right) \times X\left(\mathbb{C}_{v}\right)$ en tirant en arrière la fonction sur $\mathbb{P}^{n}\left(\mathbb{C}_{v}\right) \times \mathbb{P}^{n}\left(\mathbb{C}_{v}\right)$ définie par

$$
d_{v}(x, y)=\frac{\max _{0 \leq i<j \leq n}\left|x_{i} y_{j}-x_{j} y_{i}\right|_{v}}{\max _{0 \leq i \leq n}\left|x_{i}\right|_{v} \max _{0 \leq j \leq n}\left|y_{j}\right|_{v}} .
$$

\subsection{Métriques sur un fibré inversible}

On considère une variété projective $X$ définie sur $K$. Pour toute place $v$ de $K$, on note $X_{v}^{\text {an }}$ l'espace analytique associé au $\mathbb{C}_{v}$-schéma $X_{\mathbb{C}_{v}}$ (au sens de Berkovich si $v$ est une place finie). Sans entrer dans les détails, mentionnons que $X_{v}^{\text {an }}$ est un espace topologique compact contenant $X\left(\mathbb{C}_{v}\right)$. La topologie induite sur $X\left(\mathbb{C}_{v}\right)$ est plus fine que la topologie de Zariski, et l'on dispose d'un morphisme d'espaces localement annelés $j_{v}: X_{v}^{\text {an }} \rightarrow X$ dont la restriction de $j_{v}$ à $X\left(\mathbb{C}_{v}\right)$ envoie chaque élément de $X\left(\mathbb{C}_{v}\right)$ sur son point correspondant dans $X$. Pour tout point $x$ de $X$, on note $\mathscr{O}_{X, x}$ l'anneau local défini par $x$ et $\mathscr{L}_{x}$ le $\mathscr{O}_{X, x}$-module des germes de $\mathscr{L}$ en $x$.

Définition 2.1. Soit $\mathscr{L}$ un fibré inversible sur $X$. Une métrique sur $\mathscr{L}$ est la donnée, pour chaque place $v$ de $K$, d'une application qui associe à chaque point $x$ de $X\left(\mathbb{C}_{v}\right)$ une fonction $\|.\|_{v}(x): \mathscr{L}_{x} \otimes_{\mathscr{O}_{X, x}} \mathbb{C}_{v} \rightarrow \mathbb{R}_{\geq 0}$ telle que pour tout ouvert $U$ de $X$ et toute section $s \in \mathrm{H}^{0}(U, \mathscr{L}) \otimes_{K} \mathbb{C}_{v}$,

1. l'application $\|s\|_{v}: x \mapsto\|s\|_{v}(x)$ s'étend en une fonction continue sur $U_{v}^{\text {an }}$ (pour la topologie induite par celle de $X_{v}^{\text {an }}$ ),

2. pour toute fonction continue $f \in \mathscr{O}_{X}(U) \otimes \mathbb{C}_{v}$ et tout $x \in U\left(\mathbb{C}_{v}\right)$,

$$
\|f s\|_{v}(x)=|f(x)|_{v}\|s\|_{v}(x)
$$

3. si $x \in U\left(\mathbb{C}_{v}\right)$ est tel que $s(x) \neq 0$, alors $\|s\|_{v}(x) \neq 0$.

Remarque 2.2. La condition de continuité 1 nous permettra de définir la norme sup d'une section de $\mathscr{L}$. Les conditions 2 et 3 impliquent que pour tout point $x$ de $X\left(\mathbb{C}_{v}\right)$, une métrique sur $\mathscr{L}$ définit une norme $\|\cdot\|_{v}(x)$ sur $\mathscr{L}_{x} \otimes \mathbb{C}_{v}$ pour chaque place $v$ de $K$ (cette norme est ultramétrique si la place est finie).

On appelle modèle de $(X, \mathscr{L})$ un $\mathscr{O}_{K}$-schéma projectif et plat $\mathfrak{X}$ muni d'un fibré inversible $\mathfrak{L}$ tel que $X \cong \mathfrak{X} \times \mathscr{O}_{K} \operatorname{Spec}(K)$ et $\mathscr{L} \cong \mathfrak{L} \otimes_{\mathscr{O}_{K}} K$. Soit $v$ une place finie de $K$; on note $\widehat{\mathscr{O}}_{v}$ l'anneau de valuation de $\mathbb{C}_{v}$. Soit $x \in X\left(\mathbb{C}_{v}\right)$ et $\widehat{x} \in \mathfrak{X}\left(\widehat{\mathscr{O}}_{v}\right)$ un point prolongeant $x: x=\widehat{x}_{\mid \text {Spec } K}$. Si $s \in \mathscr{L}_{x} \otimes \mathbb{C}_{v}$, on pose

$$
\|s\|_{\mathfrak{L}, v}(x)=\inf \left\{|t|_{v} \mid t \in \mathbb{C}_{v}^{\times}, t^{-1} s \in \widehat{x}^{*} \mathfrak{L}\right\} .
$$

Cette définition définit une application $\|\cdot\|_{\mathfrak{L}, v}$ qui satisfait aux conditions de la définition 2.1 . 
Définition 2.3. Soit $\overline{\mathscr{L}}=\left(\mathscr{L},\left(\|\cdot\|_{v}\right)_{v \in \Sigma_{K}}\right)$ la donnée d'un fibré inversible $\mathscr{L}$ sur $X$ muni d'une métrique $\left(\|\cdot\|_{v}\right)_{v \in \Sigma_{K}}$. On dit que $\overline{\mathscr{L}}$ est un fibré inversible adélique si la métrique satisfait aux conditions suivantes :

1. pour toute place $v \in \Sigma_{K},\|\cdot\|_{v}$ est invariante par l'action du groupe de Galois $\operatorname{Gal}\left(\mathbb{C}_{v} / K_{v}\right)$;

2. il existe un modèle $(\mathfrak{X}, \mathfrak{L})$ de $(X, \mathscr{L})$ tel que $\|\cdot\|_{v}=\|\cdot\|_{\mathfrak{L}, v}$ pour toute place $v \in \Sigma_{K, f}$ en dehors d'un nombre fini.

Une telle métrique est appelée métrique adélique. Dans la suite, on notera $\|s(x)\|_{v}$ plutôt que $\|s\|_{v}(x)$.

Exemple 2.4. Soit $\overline{\mathscr{L}}$ un fibré inversible très ample sur $X$ et soit $\left(s_{1}, \ldots, s_{\ell}\right)$ une base de $\mathrm{H}^{0}(X, \mathscr{L})$. Pour toute place $v$ de $K$, tout $x \in X\left(\mathbb{C}_{v}\right)$ et toute section $s \in \mathrm{H}^{0}(X, \mathscr{L}) \backslash\{0\}$ ne s'annulant pas en $x$, on pose

$$
\|s(x)\|_{v}=\left(\max _{0 \leq i \leq \ell}\left|\frac{s_{i}(x)}{s(x)}\right|_{v}\right)^{-1} .
$$

La métrique $\left(\|\cdot\|_{v}\right)_{v \in \Sigma_{K}}$ ainsi définie est adélique.

$\operatorname{Si}\left(\mathscr{L}_{1},\left(\|\cdot\|_{1, v}\right)_{v \in \Sigma_{K}}\right)$ et $\left(\mathscr{L}_{2},\left(\|\cdot\|_{2, v}\right)_{v \in \Sigma_{K}}\right)$ sont deux fibrés inversibles munis de métriques sur $X$, il est possible de définir une métrique produit sur $\mathscr{L}_{1} \otimes \mathscr{L}_{2}$ de la façon suivante. Pour toute place $v \in \Sigma_{K}$, pour tout $x \in X\left(\mathbb{C}_{v}\right)$ et toutes sections $s_{1}$ de $\mathscr{L}_{1} \otimes \mathbb{C}_{v}$ et $s_{2}$ de $\mathscr{L}_{2} \otimes \mathbb{C}_{v}$ définies au voisinage de $x$, on pose

$$
\left\|s_{1} \otimes s_{2}(x)\right\|_{v}=\left\|s_{1}(x)\right\|_{v}\left\|s_{2}(x)\right\|_{v} .
$$

Si de plus $\left(\|\cdot\|_{1, v}\right)_{v \in \Sigma_{K}}$ et $\left(\|\cdot\|_{2, v}\right)_{v \in \Sigma_{K}}$ sont adéliques, alors $\left(\|\cdot\|_{v}\right)_{v \in \Sigma_{K}}$ est une métrique adélique.

Définition 2.5. Soit $L$ une extension de $K$ et $x$ un point de $X(L)$. Soit $\overline{\mathscr{L}}=$ $\left(\mathscr{L},\left(\|\cdot\|_{v}\right)_{v \in \Sigma_{L}}\right)$ un fibré inversible adélique et $s$ une section locale de $\mathscr{L}$ telle que $s(x) \neq 0$. On définit la hauteur (absolue) de $x$ relativement à $\mathscr{L}$ comme

$$
h_{\overline{\mathscr{L}}}(x)=-\sum_{v \in \Sigma_{L}} \frac{\left[L_{v}: \mathbb{Q}_{v}\right]}{[L: \mathbb{Q}]} \log \|s(x)\|_{v}
$$

Cette définition ne dépend pas de la section $s$ choisie (par la formule du produit) ni du corps $L$. En effet, si $L^{\prime}$ est une extension finie de $L$, alors

$$
\begin{aligned}
h_{\bar{L}}(x) & =-\sum_{v \in \Sigma_{L}} \frac{\left[L_{v}: \mathbb{Q}_{v}\right]}{[L: \mathbb{Q}]} \log \|s(x)\|_{v} \\
& =-\sum_{v \in \Sigma_{L}} \sum_{w \in \Sigma_{L}^{\prime}, w \mid v} \frac{\left[L_{w}^{\prime}: L_{v}\right]\left[L_{v}: \mathbb{Q}_{v}\right]}{\left[L^{\prime}: L\right][L: \mathbb{Q}]} \log \|s(x)\|_{v} \\
& =-\sum_{w \in \Sigma_{L}^{\prime}} \frac{\left[L_{w}^{\prime}: \mathbb{Q}_{w}\right]}{\left[L^{\prime}: \mathbb{Q}\right]} \log \|s(x)\|_{w} .
\end{aligned}
$$

Dans la suite, nous noterons $h_{\mathscr{L}}$ au lieu de $h_{\overline{\mathscr{L}}}$ s'il n'y a pas d'ambiguïté sur le choix de la structure adélique. 
Exemple 2.6. Soit $\left(s_{0}, \ldots, s_{n}\right)$ une base de $\mathrm{H}^{0}\left(\mathbb{P}_{K}^{n}, \mathscr{O}(1)\right)$ correspondant à un choix de coordonnées homogènes. On munit le fibré $\mathscr{O}(1)$ de la métrique de l'exemple 2.4 associée à cette base : pour toute place $v$ de $K$, tout $x \in \mathbb{P}^{n}\left(\mathbb{C}_{v}\right)$ et toute section $s \in \mathrm{H}^{0}\left(\mathbb{P}_{K}^{n}, \mathscr{O}(1)\right) \backslash\{0\}$ ne s'annulant pas en $x$,

$$
\|s(x)\|_{v}=\left(\max _{0 \leq i \leq n}\left|\frac{s_{i}(x)}{s(x)}\right|_{v}\right)^{-1} .
$$

Alors pour tout point $x=\left(x_{0}: \cdots: x_{n}\right) \in \mathbb{P}^{n}(K)$,

$$
h_{\overline{\mathscr{O}(1)}}(x)=\sum_{v \in \Sigma_{K}} \frac{\left[K_{v}: \mathbb{Q}_{v}\right]}{[K: \mathbb{Q}]} \log \max \left\{\left|x_{0}\right|_{v}, \ldots,\left|x_{n}\right|_{v}\right\}=h(x) .
$$

Si l'on considère la métrique donnée par

$$
\|s(x)\|_{v}=\left(\left|\left(s_{i}(x) / s(x)\right)_{0 \leq i \leq n}\right|_{2, v}\right)^{-1}
$$

pour toute place $v$, alors

$$
h_{\overline{\mathscr{O}(1)}}(x)=\sum_{v \in \Sigma_{K}} \frac{\left[L_{v}: \mathbb{Q}_{v}\right]}{[L: \mathbb{Q}]} \log \left|\left(x_{0}, \ldots, x_{n}\right)\right|_{2, v}=h_{2}(x) .
$$

On retrouve les hauteurs projectives définies au paragraphe 2.1.

Notons $V=\mathrm{H}^{0}(X, \mathscr{L})$ l'espace vectoriel des sections globales de $\mathscr{L}$. La structure de fibré inversible adélique de $\mathscr{L}$ induit une structure d'espace vectoriel normé sur $V \otimes_{K} \mathbb{C}_{v}$ pour toute place $v \in \Sigma_{K}$ en définissant la norme

$$
\|s\|_{v, \text { sup }}=\sup _{x \in X\left(\mathbb{C}_{v}\right)}\|s(x)\|_{v}
$$

( $V$ est même muni d'une structure de fibré vectoriel adélique, au sens de [8]). Cette quantité est finie étant donné que $X\left(\mathbb{C}_{v}\right)$ est contenu dans le compact $X^{\text {an }}$ et que $\|s\|: x \mapsto\|s(x)\|_{v}$ est continue. On définit la hauteur d'une section par rapport à cette norme :

$$
h_{\mathscr{L}}(s)=\sum_{v \in M_{K}} \frac{\left[K_{v}: \mathbb{Q}_{v}\right]}{[K: \mathbb{Q}]} \log \|s\|_{v, \text { sup }},
$$

que nous noterons plus simplement $h(s)$ s'il n'y a pas d'ambiguïté.

\subsection{Minimum absolu et dernier minimum}

Soit $X$ une variété projective définie sur $K$.

Définition 2.7. Soit $\overline{\mathscr{L}}$ un fibré inversible adélique sur $X$. On définit le dernier minimum de $\overline{\mathscr{L}}$ comme la quantité

$$
\lambda_{\max }(\overline{\mathscr{L}})=\min \left\{\max _{i \in I} h_{\mathscr{L}}\left(s_{i}\right) \mid\left(s_{i}\right)_{i \in I} \text { est une base de } \mathrm{H}^{0}(X, \mathscr{L})\right\} .
$$


La quantité $\lambda_{\max }(\overline{\mathscr{L}})$ est le logarithme du dernier minimum de $\mathrm{H}^{0}(X, \mathscr{L})$ au sens de Roy et Thunder [18].

Soit $\overline{\mathscr{L}}=\left(\mathscr{L},\left(\|\cdot\|_{v}\right)_{v \in \Sigma_{K}}\right)$ un fibré inversible adélique sur $X$. On note $\mathbf{B}(\mathscr{L})$ le lieu de base stable de $\mathscr{L}$, c'est-à-dire l'intersection des lieux de base $\mathscr{B}(m \mathscr{L})$ des fibrés $m \mathscr{L}$ pour $m \in \mathbb{N}$ (cette définition s'étend naturellement aux éléments de $\operatorname{Pic}_{\mathbb{Q}}(X)=\operatorname{Pic}(X) \otimes_{\mathbb{Z}} \mathbb{Q}$, voir la remarque 2.1.24 de [11]). D'après la proposition 2.1.21 de [11], il existe un entier $m_{0}$ tel que pour tout entier $m$ multiple de $m_{0}, \mathbf{B}(\mathscr{L})=\mathscr{B}(m \mathscr{L})$. Soit $z \in(X \backslash \mathbf{B}(\mathscr{L}))(\overline{\mathbb{Q}})$ et soit $\left(s_{i}\right)_{i \in I}$ une base de $\mathrm{H}^{0}\left(X, m_{0} \mathscr{L}\right)$. Comme $z \notin \mathscr{B}\left(m_{0} \mathscr{L}\right)$, il existe $i \in I$ tel que $s_{i}(z) \neq 0$. On en déduit que

$$
\begin{aligned}
h_{\bar{L}}(z)=\frac{1}{m_{0}} h_{\overline{m_{0} \mathscr{L}}}(z) & =-\frac{1}{m_{0}} \sum_{v \in \Sigma_{K}} \frac{\left[K_{v}: \mathbb{Q}_{v}\right]}{[K: \mathbb{Q}]} \log \left\|s_{i}(z)\right\|_{v} \\
& \geq-\frac{1}{m_{0}} h_{m_{0} \mathscr{L}}\left(s_{i}\right) \geq-\frac{1}{m_{0}} \max _{i \in I} h_{m_{0} \mathscr{L}}\left(s_{i}\right) .
\end{aligned}
$$

Ce raisonnement est valable pour toute base $\mathrm{de}^{0}\left(X, m_{0} \mathscr{L}\right)$ et tout $z \in(X \backslash$ $\mathbf{B}(\mathscr{L}))(\overline{\mathbb{Q}})$. On en déduit que la quantité

$$
\inf \left\{h_{\bar{L}}(z) \mid z \in(X \backslash \mathbf{B}(\mathscr{L}))(\overline{\mathbb{Q}})\right\}
$$

est finie. Si le fibré $\mathscr{L}$ est semi-ample (c'est-à-dire qu'il existe $m_{0} \in \mathbb{N}$ tel que pour tout $m \geq m_{0}$, le fibré $m \mathscr{L}$ est engendré par ses sections globales), alors $\mathbf{B}(\mathscr{L})=\emptyset$. Cette observation justifie la définition suivante.

Définition 2.8. Si $\overline{\mathscr{L}}=\left(\mathscr{L},\left(\|\cdot\|_{v}\right)_{v \in \Sigma_{K}}\right)$ est un fibré inversible adélique sur $X$ avec $\mathscr{L}$ semi-ample, on définit le minimum absolu de $\overline{\mathscr{L}}$ comme le nombre réel

$$
\mathrm{e}(\overline{\mathscr{L}})=\inf _{z \in X(\overline{\mathbb{Q}})} h_{\overline{\mathscr{L}}}(z) .
$$

D'après les remarques précédentes, on a le lemme suivant.

Lemme 2.9. Soit $\overline{\mathscr{L}}=\left(\mathscr{L},\left(\|\cdot\|_{v}\right)_{v \in \Sigma_{K}}\right)$ un fibré inversible adélique sur $X$. Alors pour tout $m \in \mathbb{N} \backslash\{0\}$ tel que $\mathbf{B}(\mathscr{L})=\mathscr{B}(m \mathscr{L})$, on a

$$
\inf \left\{h_{\overline{\mathscr{L}}}(z) \mid z \in(X \backslash \mathbf{B}(\mathscr{L}))(\overline{\mathbb{Q}})\right\} \geq-\frac{1}{m} \lambda_{\max }(\overline{m \mathscr{L}}) .
$$

En particulier, si $\mathscr{L}$ est semi-ample alors pour tout $m \in \mathbb{N} \backslash\{0\}$ tel que $m \mathscr{L}$ est engendré par ses sections globales, l'inégalité

$$
\mathrm{e}(\overline{\mathscr{L}}) \geq-\frac{1}{m} \lambda_{\max }(\overline{m \mathscr{L}})
$$

est vérifiée.

La proposition suivante est une conséquence d'un résultat classique de Zhang [20] et permet de comparer le comportement asymptotique du dernier minimum et le minimum absolu d'un fibré ample équipé d'une métrique semi-positive (au sens de $[16, \S 1.12]$ et $[20, \S 1,(1.1)])$.

Proposition 2.10. Soit $\overline{\mathscr{L}}=\left(\mathscr{L},\left(\|\cdot\|_{v}\right)_{v \in \Sigma_{K}}\right)$ un fibré inversible adélique sur $X$. On suppose que la métrique sur $\mathscr{L}$ est semi-positive et que $\mathscr{L}$ est ample. Alors

$$
-\mathrm{e}(\overline{\mathscr{L}})=\lim _{m \rightarrow+\infty} \frac{1}{m} \lambda_{\max }(\overline{m \mathscr{L}}) .
$$


Démonstration : D'après le lemme 2.9 , si $m \in \mathbb{N}$ est suffisamment grand on a l'inégalité

$$
\mathrm{e}(\overline{\mathscr{L}}) \geq-\frac{1}{m} \lambda_{\max }(\overline{m \mathscr{L}})
$$

On en déduit que

$$
\liminf _{m \rightarrow+\infty} \frac{1}{m} \lambda_{\max }(\overline{m \mathscr{L}}) \geq-\mathrm{e}(\overline{\mathscr{L}})
$$

Soit $\varepsilon>0$ et $\alpha=-\mathrm{e}(\overline{\mathscr{L}})+\varepsilon$. On définit une nouvelle structure de fibré adélique $\overline{\mathscr{L}(\alpha)}=\left(\mathscr{L},\left(\|\cdot\|_{\alpha, v}\right)_{v \in \Sigma_{K}}\right)$ sur $\mathscr{L}$ en posant $\|\cdot\|_{\alpha, v}=e^{-\alpha}\|\cdot\|_{v}$ si $v$ est archimédienne et $\|\cdot\|_{\alpha, v}=\|\cdot\|_{v}$ sinon. Pour tout $z \in X(\overline{\mathbb{Q}})$, on a ainsi

$$
h \overline{\mathscr{L}(-\alpha)}(z)=h_{\overline{\mathscr{L}}}(z)+\alpha \geq \varepsilon>0 .
$$

D'après le théorème 0.2 de [17], l'hypothèse du corollaire 5.7 (2) de [20] est satisfaite et on obtient : si $m \in \mathbb{N}$ est suffisamment grand il existe une base $\left(s_{i}\right)_{i \in I}$ de $\mathrm{H}^{0}(X, m \mathscr{L})$ telle que pour tout $i \in I$,

$$
h_{m \mathscr{L}(\alpha)}\left(s_{i}\right)=h_{m \mathscr{L}}\left(s_{i}\right)-m \alpha<0 .
$$

On en déduit que

$$
\limsup _{m \rightarrow+\infty} \frac{1}{m} \lambda_{\max }(\overline{m \mathscr{L}}) \leq \alpha=-\mathrm{e}(\overline{\mathscr{L}})+\varepsilon .
$$

En faisant tendre $\varepsilon$ vers 0 , on en déduit que

$$
\liminf _{m \rightarrow+\infty} \frac{1}{m} \lambda_{\max }(\overline{m \mathscr{L}})=\limsup _{m \rightarrow+\infty} \frac{1}{m} \lambda_{\max }(\overline{m \mathscr{L}})=-\mathrm{e}(\overline{\mathscr{L}})
$$

ce qui démontre la proposition.

\section{L'approche de McKinnon et Roth}

Dans cette partie, nous montrons comment adapter la démonstration du théorème 3.3 de McKinnon et Roth présentée dans l'article [14] pour obtenir un résultat effectif. Soit $X$ une variété projective définie sur un corps de nombres $K, L$ une extension finie de $K, v$ une place de $L$ et $x \in X(L)$ un point fermé. Soit $\mathscr{L}$ un fibré inversible nef sur $X$. On note $X_{L}=X \times{ }_{K} \operatorname{Spec}(L), \pi: \widetilde{X} \rightarrow X_{L}$ l'éclatement de $X_{L}$ en $x$ et $E$ le diviseur exceptionnel associé. Soit $\varphi: X \hookrightarrow \mathbb{P}_{K}^{n}$ un plongement et $d_{v}$ la distance associée (définie dans la partie 2.2). Rappelons que la constante de Seshadri $\epsilon_{x}(\mathscr{L})$, définie par Demailly en 1990 (voir $[5, \S 6]$ ), est égale à la quantité

$$
\epsilon_{x}(\mathscr{L})=\sup \left\{\gamma \in \mathbb{Q} \mid \pi^{*} \mathscr{L}-\gamma E \text { est nef }\right\}
$$

Dans la suite, pour tout rationnel positif $\gamma$, on notera $\mathscr{L}_{\gamma}=\pi^{*} \mathscr{L}-\gamma E \epsilon$ $\operatorname{Pic}_{\mathbb{Q}}(\tilde{X})=\operatorname{Pic}(\tilde{X}) \otimes_{\mathbb{Z}} \mathbb{Q}$. On peut associer à $\mathscr{L}$ une fonction de hauteur comme dans le théorème B.3.2 de [9].

On note $\widetilde{\mathbb{P}}_{L}^{n} \subset \mathbb{P}_{L}^{n} \times \mathbb{P}_{L}^{n-1}$ l'éclatement de $\mathbb{P}_{L}^{n}$ en $\varphi(x)$ et $E^{\prime}$ le diviseur exceptionnel. Le fibré inversible $\mathscr{O}_{\widetilde{\mathbb{P}}_{L}^{n}}\left(E^{\prime}\right)$ est la restriction de $\mathscr{O}_{\mathbb{P}_{L}^{n} \times \mathbb{P}_{L}^{n-1}}(1,-1)$ à $\widetilde{\mathbb{P}}_{L}^{n}$. 
On munit $\mathscr{O}_{\mathbb{P}_{L}^{n}}(1)$ et $\mathscr{O}_{\mathbb{P}_{L}^{n-1}}(1)$ de métriques adéliques de sorte que $h_{\mathscr{O}_{\mathbb{P}}^{n}(1)}(\alpha)=$ $h_{2}(\alpha)$ et $h_{\mathscr{O}_{\mathbb{P} n-1}(1)}(\beta)=h_{2}(\beta)$ pour tous $\alpha \in \mathbb{P}_{L}^{n}(\bar{K}), \beta \in \mathbb{P}_{L}^{n-1}(\bar{K})$ (voir l'exemple 2.6). Ce choix induit une métrique sur $\mathscr{O}_{\mathbb{P}_{L}^{n} \times \mathbb{P}_{L}^{n-1}}(1,-1)$ telle que

$$
h_{\mathscr{O}(1,-1)}(\alpha, \beta)=h_{2}(\alpha)-h_{2}(\beta)
$$

pour tout $(\alpha, \beta) \in\left(\mathbb{P}^{n} \times \mathbb{P}^{n-1}\right)(\bar{K})=\mathbb{P}^{n}(\bar{K}) \times \mathbb{P}^{n-1}(\bar{K})$. Notons $\varphi(x)=\left(x_{0}\right.$ : $\left.\cdots: x_{n}\right)$, et supposons que $x_{0} \neq 0$. Avec ces notations, $\left(\widetilde{\mathbb{P}}_{L}^{n} \backslash E^{\prime}\right)(L)$ est l'ensemble

$$
\left\{\left(\left(\alpha_{0}: \cdots: \alpha_{n}\right) ;\left(x_{0} \alpha_{1}-x_{1} \alpha_{0}: \cdots: x_{0} \alpha_{n}-x_{n} \alpha_{0}\right)\right) \in \mathbb{P}^{n}(L) \times \mathbb{P}^{n-1}(L)\right\} .
$$

Par la propriété universelle de l'éclatement, il existe un morphisme $\widetilde{\varphi}: \widetilde{X} \rightarrow \widetilde{\mathbb{P}}_{L}^{n}$ tel que $\mathscr{O}_{\widetilde{X}}(E)=\widetilde{\varphi}^{*} \mathscr{O}_{\widetilde{\mathbb{P}}_{L}^{n}}\left(E^{\prime}\right)$. On obtient une métrique adélique sur $\mathscr{O}_{\widetilde{X}}(E)$ en tirant en arrière celle de $\mathscr{O}_{\mathbb{P}_{L}^{n} \times \mathbb{P}_{L}^{n-1}}(1,-1)$. Pour tout $z \in(\widetilde{X} \backslash E)(L)$, on a

$$
h_{E}(z):=h \overline{\mathscr{O}(E)}(z)=\sum_{w \in \Sigma_{L}} \frac{\left[L_{w}: \mathbb{Q} w\right]}{[L: \mathbb{Q}]} \log \left(\frac{\left|\left(z_{0}, \ldots, z_{n}\right)\right|_{2, w}}{\left|\left(x_{0} \alpha_{j}-x_{j} \alpha_{0}\right)_{1 \leq j \leq n}\right|_{2, w}}\right),
$$

où $\varphi(\pi(z))=\left(z_{0}: \cdots: z_{n}\right)$. On en déduit que

$$
\begin{aligned}
h_{E}(z)+h_{2}(\varphi(x)) & =\sum_{w \in \Sigma_{L}} \frac{\left[L_{w}: \mathbb{Q}_{w}\right]}{[L: \mathbb{Q}]} \log \left(\frac{\left|\left(z_{0}, \ldots, z_{n}\right)\right|_{2, w}\left|\left(x_{0}, \ldots, x_{n}\right)\right|_{2, w}}{\left|\left(x_{0} \alpha_{j}-x_{j} \alpha_{0}\right)_{1 \leq j \leq n}\right|_{2, w}}\right) \\
& \geq-\frac{\left[L_{v}: \mathbb{Q} v\right]}{[L: \mathbb{Q}]} \log d_{v}(x, \pi(z)) .
\end{aligned}
$$

Soit $\gamma$ un nombre rationnel strictement positif tel que $\mathscr{L}_{\gamma}$ soit dans le cône effectif de $\operatorname{Pic}_{\mathbb{Q}}(\widetilde{X})$. Soit $m$ un entier non nul tel que $m \gamma \in \mathbb{N}$. Considérons un point $y \in X \backslash\left(\pi\left(\mathbf{B}\left(\mathscr{L}_{\gamma}\right)\right)\right)(K)$. D'après le théorème B.3.2 de [9] et la proposition 2.1.21 de [11], il existe une constante $c_{\gamma}$ telle que $h_{m} \mathscr{L}_{\gamma}(y) \geq c_{\gamma}$. Si $y \neq x$, on a ainsi

$$
\begin{aligned}
c_{\gamma}<h_{m \mathscr{L}_{\gamma}}(y) & =m h_{\mathscr{L}}(y)-m \gamma h_{E}(y) \\
& \leq m h_{\mathscr{L}}(y)+m \gamma \frac{\left[L_{v}: \mathbb{Q}_{v}\right]}{[L: \mathbb{Q}]} \log d_{v}(x, y)+m \gamma h_{2}(\varphi(x)),
\end{aligned}
$$

d'où

$$
\log \left(d_{v}(x, y)\right)>\frac{[L: \mathbb{Q}]}{m \gamma\left[L_{v}: \mathbb{Q}_{v}\right]} c_{\gamma}-\frac{[L: \mathbb{Q}]}{\left[L_{v}: \mathbb{Q}_{v}\right]} h_{2}(\varphi(x))-\frac{[L: \mathbb{Q}]}{\gamma\left[L_{v}: \mathbb{Q}_{v}\right]} h_{\mathscr{L}}(y),
$$

ce qui démontre le théorème 1.2. Pour obtenir une version effective de ce théorème, il suffit d'expliciter la constante $c_{\gamma}$. Comme le souligne la remarque B.3.2.1 (ii) de [9], la constante $c_{\gamma}$ est en général difficile à minorer en pratique. Cependant, si le fibré $\mathscr{L}$ est adélique, on a l'inégalité $h_{m \mathscr{L}_{\gamma}}(y) \geq \inf \left\{h_{m \mathscr{L}_{\gamma}}(z) \mid z \in\right.$ $\left(\tilde{X} \backslash \mathbf{B}\left(\mathscr{L}_{\gamma}\right)(K)\right\}$ pour tout point $y \in X \backslash\left(\pi\left(\mathbf{B}\left(\mathscr{L}_{\gamma}\right)\right)\right)(K)$ et l'on peut donc prendre

$$
\begin{aligned}
c_{\gamma} & =\inf \left\{h_{m \mathscr{L}_{\gamma}}(z) \mid z \in\left(\widetilde{X} \backslash \mathbf{B}\left(\mathscr{L}_{\gamma}\right)\right)(K)\right\} \\
& =m \inf \left\{h_{\mathscr{L}}(\pi(z))-\gamma h_{E}(z) \mid z \in\left(\widetilde{X} \backslash \mathbf{B}\left(\mathscr{L}_{\gamma}\right)\right)(K)\right\}
\end{aligned}
$$

(voir aussi la remarque 3.2 ci-dessous). Nous avons donc démontré le théorème suivant. 
Théorème 3.1. Soit $\mathscr{L}$ un fibré inversible nef muni d'une métrique adélique et $\gamma>0$ un nombre rationnel tel que $\mathscr{L}_{\gamma}$ soit dans le cône effectif de $\operatorname{Pic}_{\mathbb{Q}}(\widetilde{X})$. Pour tout point $y \in X(K)$ distinct de $x$ tel que $y \notin \pi\left(\mathbf{B}\left(\mathscr{L}_{\gamma}\right)\right.$ ), l'inégalité suivante est vérifiée :

$$
\log \left(d_{v}(x, y)\right) \geq \frac{[L: \mathbb{Q}]}{\gamma\left[L_{v}: \mathbb{Q}_{v}\right]}\left(C_{\gamma}-h_{\mathscr{L}}(y)\right)
$$

ò̀

$$
C_{\gamma}=\inf \left\{h_{\mathscr{L}}(\pi(z))-\gamma h_{E}(z) \mid z \in\left(\tilde{X} \backslash \mathbf{B}\left(\mathscr{L}_{\gamma}\right)\right)(K)\right\}-\gamma h_{2}(\varphi(x)) .
$$

Ce théorème implique le corollaire 1.5 de la façon suivante. Si $\mathscr{L}$ est ample, alors on sait que $\epsilon_{x}(\mathscr{L})>0$ et pour tout nombre réel strictement positif $\gamma<$ $\epsilon_{x}(\mathscr{L}), \mathscr{L}_{\gamma}$ est ample (voir la discussion qui suit la définition 2.9 de [14]). On en déduit que le lieu de base stable $\mathbf{B}\left(\mathscr{L}_{\gamma}\right)$ du théorème 3.1 est vide. Soit $\varepsilon>0$ et soit $y \in X(K)$ un point distinct de $x$. Si $\gamma<\epsilon_{x}(\mathscr{L})$ est suffisamment proche de $\epsilon_{x}(\mathscr{L})$ et si $h_{\mathscr{L}}(y) \geq 0$, on a l'implication

$$
\begin{aligned}
& \log \left(d_{v}(x, y)\right)<-\left(\frac{[L: \mathbb{Q}]}{\epsilon_{x}(\mathscr{L})\left[L_{v}: \mathbb{Q}_{v}\right]}+\varepsilon\right) h_{\mathscr{L}}(y) \\
& \Longrightarrow \log \left(d_{v}(x, y)\right)<-\left(\frac{[L: \mathbb{Q}]}{\gamma\left[L_{v}: \mathbb{Q}_{v}\right]}+\frac{\varepsilon}{2}\right) h_{\mathscr{L}}(y) .
\end{aligned}
$$

On obtient le corollaire 1.5 en posant

$$
b(x, \mathscr{L})=-2 \frac{[L: \mathbb{Q}]}{\left[L_{v}: \mathbb{Q}_{v}\right]} \min \left\{0, C_{\gamma} / \gamma\right\} .
$$

La propriété de Northcott (voir [16, théorème 9.11]) implique alors qu'il n'existe qu'un nombre fini de points $y \in X(K)$ distincts de $x$ tels que

$$
\log \left(d_{v}(x, y)\right)<-\left(\frac{[L: \mathbb{Q}]}{\epsilon_{x}(\mathscr{L})\left[L_{v}: \mathbb{Q}_{v}\right]}+\varepsilon\right) h_{\mathscr{L}}(y) .
$$

La minoration du théorème dépend fortement du choix de métrique sur $E$ (à cause du terme $\left.\inf \left\{h_{\mathscr{L}}(\pi(z))-\gamma h_{E}(z) \mid z \in\left(\widetilde{X} \backslash \mathbf{B}\left(\mathscr{L}_{\gamma}\right)\right)(K)\right\}\right)$. L'objectif de la suite du texte est d'obtenir des bornes indépendantes d'un tel choix. Nous allons utiliser une autre approche, inspirée de celle de [13], où les auteurs utilisent le théorème 1.1 de Faltings et Wüstholz rappelé dans l'introduction pour généraliser le théorème de Roth. Dans le cas où l'on ne s'intéresse qu'à une seule place (card $\mathscr{P}=1)$, nous verrons comment montrer facilement une version effective de cet énoncé dans la partie 4 . Nous appliquerons ce résultat pour obtenir une version effective du théorème de Liouville, qui fournit une borne qui ne dépend pas d'un choix de métriques sur $E$ dans le cas où le point $x$ est régulier.

Remarque 3.2. D'après le lemme 2.9, la quantité inf $\left\{h_{\mathscr{L}}(\pi(z))-\gamma h_{E}(z) \mid z \in\right.$ $\left.\left(\tilde{X} \backslash \mathbf{B}\left(\mathscr{L}_{\gamma}\right)\right)(K)\right\}$ du théorème 3.1 est minorée par la quantité

$-\frac{1}{r} \lambda_{\max }\left(\overline{r \mathscr{L}_{\gamma}}\right)=-\frac{1}{r} \min \left\{\max _{1 \leq i \leq t} h_{r \mathscr{L}_{\gamma}}\left(s_{i}\right) \mid\left(s_{1}, \ldots, s_{t}\right)\right.$ base de $\left.H^{0}\left(X_{L}, r \mathscr{L}_{\gamma}\right)\right\}$, 
où $r$ est entier tel que $r \gamma \in \mathbb{N} \backslash\{0\}$ et tel que $\mathscr{B}\left(r \mathscr{L}_{\gamma}\right)=\mathbf{B}\left(\mathscr{L}_{\gamma}\right)$. Mais ici la quantité $\frac{1}{r} \lambda_{\max }\left(\overline{r \mathscr{L}_{\gamma}}\right)$ dépend encore du choix de métriques sur $E$. Dans la partie 5 , nous verrons comment remplacer cette quantité $\frac{1}{r} \lambda_{\max }\left(\overline{r \mathscr{L}_{\gamma}}\right)$ par

$$
\frac{1}{r} \min \left\{\max _{i \in I} h_{\mathscr{L}}\left(s_{i}\right) \mid\left(s_{i}\right)_{i \in I} \text { base de } F_{r}\right\},
$$

où $F_{r}$ est le sous-espace vectoriel de $\mathrm{H}^{0}\left(X_{L}, \mathscr{L}\right)$ des sections s'annulant à l'ordre $r \gamma$ en $x$ (corollaire 5.5).

\section{Autour du théorème de Faltings et Wüstholz}

Soit $K \subseteq L$ deux corps de nombres et soit $\mathscr{P}$ un sous-ensemble fini de $\Sigma_{L}$. On considère une variété projective $X$ sur Spec $K$ munie d'un faisceau inversible adélique $\overline{\mathscr{L}}=\left(\mathscr{L},\left(\|\cdot\|_{v}\right)_{v \in \Sigma_{K}}\right)$. Notons $V=\mathrm{H}^{0}(X, \mathscr{L})$ et $V_{L}=V \otimes L$. Soit $F$ un sous-espace vectoriel de $V_{L}$. Pour chaque place $w \in \mathscr{P}$, on fixe un nombre réel $t_{w}$. On considère le lieu de base de $F$ défini par

$$
\mathscr{B}(F)=\{x \in X \mid s(x)=0 \forall s \in F\}
$$

et on fixe $\left(s_{1}, \ldots, s_{\operatorname{dim} F}\right)$ une base de $F$.

Théorème 4.1. Pour chaque place $w$ de $\mathscr{P}$, soit $C_{w}>0$ un nombre réel. Si $\delta:=\sum_{w \in \mathscr{P}} \frac{\left[L_{w}: \mathbb{Q}_{w}\right]}{[L: \mathbb{Q}]} t_{w}>1$, alors tous les points $x \in(X \backslash \mathscr{B}(F))(K)$ satisfaisant le système d'inégalités

$$
\frac{\left\|s_{j}(x)\right\|_{w}}{\left\|s_{j}\right\|_{w, \text { sup }}} \leq C_{w} \exp \left(-t_{w} h_{\mathscr{L}}(x)\right), \quad \forall 1 \leq j \leq \operatorname{dim} F, \quad \forall w \in \mathscr{P}
$$

vérifient

$$
h_{\mathscr{L}}(x) \leq \frac{1}{\delta-1}\left(\sum_{w \in \mathscr{P}} \frac{\left[L_{w}: \mathbb{Q}_{w}\right]}{[L: \mathbb{Q}]} \log \left(C_{w}\right)+\max _{1 \leq j \leq \operatorname{dim} F} h\left(s_{j}\right)\right) .
$$

En particulier, ces points sont en nombre fini si $\mathscr{L}$ est ample.

Démonstration : Soit $x \in(X \backslash \mathscr{B}(F))(K)$ vérifiant (5). Il existe un entier $i$, $1 \leq i \leq \operatorname{dim} F$ tel que $s_{i}(x) \neq 0($ car sinon $x \in \mathscr{B}(F)$ ). Pour toute place $v$ de $L$,

$$
\left\|s_{i}(x)\right\|_{v} /\left\|s_{i}\right\|_{v, \text { sup }} \leq 1
$$

et par hypothèse

$$
\frac{\left\|s_{i}(x)\right\|_{w}}{\left\|s_{i}\right\|_{w, \text { sup }}} \leq C_{w} \exp \left(-t_{w} h_{\mathscr{L}}(x)\right)
$$

pour toute place $w \in \mathscr{P}$. On en déduit que

$$
\begin{aligned}
-\left(h_{\mathscr{L}}(x)+h\left(s_{i}\right)\right) & =\sum_{v \in \Sigma_{L}} \frac{\left[L_{v}: \mathbb{Q}_{v}\right]}{[L: \mathbb{Q}]} \log \left(\frac{\left\|s_{i}(x)\right\|_{v}}{\left\|s_{i}\right\|_{v, \text { sup }}}\right) \\
& \leq-\sum_{w \in \mathscr{P}} \frac{\left[L_{w}: \mathbb{Q} w\right]}{[L: \mathbb{Q}]} t_{w} h_{\mathscr{L}}(x)+\sum_{w \in \mathscr{P}} \frac{\left[L_{w}: \mathbb{Q}_{w}\right]}{[L: \mathbb{Q}]} \log \left(C_{w}\right),
\end{aligned}
$$


d'où

$$
(\delta-1) h_{\mathscr{L}}(x) \leq \max \left\{h\left(s_{j}\right) \mid 1 \leq j \leq \operatorname{dim} F\right\}+\sum_{w \in \mathscr{P}} \frac{\left[L_{w}: \mathbb{Q} w\right]}{[L: \mathbb{Q}]} \log \left(C_{w}\right) .
$$

On en déduit la majoration voulue grâce à l'hypothèse $\delta>1$. Si $\mathscr{L}$ est ample, on en déduit que les points vérifiant (5) sont en nombre fini d'après la propriété de Northcott (voir [16, théorème 9.11]).

On obtient immédiatement le corollaire suivant :

Corollaire 4.2. Pour chaque place $w$ de $\mathscr{P}$, soit $C_{w}>0$ un nombre réel. Si $\delta:=\sum_{w \in \mathscr{P}} \frac{\left[L_{w}: \mathbb{Q}_{w}\right]}{[L: \mathbb{Q}]} t_{w}>1$, alors tous les points $x \in(X \backslash \mathscr{B}(F))(K)$ satisfaisant le système d'inégalités

$$
\frac{\|s(x)\|_{w}}{\|s\|_{w, \text { sup }}} \leq C_{w} \exp \left(-t_{w} h_{\mathscr{L}}(x)\right), \quad \forall s \in F \backslash\{0\}, \quad \forall w \in \mathscr{P}
$$

vérifient

$$
\begin{aligned}
h_{\mathscr{L}}(x) \leq \frac{1}{\delta-1}( & \sum_{w \in \mathscr{P}} \frac{\left[L_{w}: \mathbb{Q} w\right]}{[L: \mathbb{Q}]} \log \left(C_{w}\right) \\
& \left.+\min \left\{\max _{1 \leq j \leq \operatorname{dim} F} h\left(s_{j}\right) \mid\left(s_{1}, \ldots, s_{\operatorname{dim} F}\right) \text { base de } F\right\}\right) .
\end{aligned}
$$

En particulier, ces points sont en nombre fini si $\mathscr{L}$ est ample.

Pour chaque place $w$ de $\mathscr{P}$, on considère une filtration séparée $\mathscr{F}_{w}$ de $V_{L}$, donnée par un drapeau

$$
V_{L}=V_{0}^{(w)} \supsetneq V_{1}^{(w)} \supsetneq \cdots \supsetneq V_{e_{w}}^{(w)} \supsetneq\{0\}
$$

et une suite finie de nombres réels

$$
c_{w, 0}<c_{w, 1}<\ldots<c_{w, e_{w}}
$$

(voir par exemple [1]). Pour tout nombre réel $t \in\left[c_{w, 0}, c_{w, e_{w}}\right]$ on définit un sous-espace vectoriel $\mathscr{F}_{w}^{t} V_{L}$ de $V_{L}$ en posant

$$
\mathscr{F}_{w}^{t} V_{L}=\bigcup_{\substack{0 \leq i \leq e_{w} \\ c_{w}, i \geq t}} V_{i}^{(w)} .
$$

Pour chaque place $w \in \mathscr{P}$, on considère un nombre réel $t_{w}$ tel que $c_{w, 0} \leq$ $t_{w} \leq c_{w, e_{w}}$. En appliquant le théorème 4.1 avec $F=\bigcap_{w \in \mathscr{P}} \mathscr{F}_{w}^{t_{w}} V_{L}$, on obtient le résultat suivant.

Corollaire 4.3. Pour chaque place $w$ de $\mathscr{P}$, soit $C_{w}>0$ un nombre réel. Si $\delta:=\sum_{w \in \mathscr{P}} \frac{\left[L_{w}: \mathbb{Q}_{w}\right]}{[L: \mathbb{Q}]} t_{w}>1$, alors tous les points $x \in(X \backslash \mathscr{B}(F))(K)$ satisfaisant le système d'inégalités

$$
\frac{\|s(x)\|_{w}}{\|s\|_{w, \text { sup }}} \leq C_{w} \exp \left(-t_{w} h_{\mathscr{L}}(x)\right), \quad \forall s \in \mathscr{F}_{w}^{t_{w}} V_{L} \backslash\{0\}, \quad \forall w \in \mathscr{P}
$$


vérifient

$$
\begin{aligned}
h_{\mathscr{L}}(x) \leq \frac{1}{\delta-1}( & \sum_{w \in \mathscr{P}} \frac{\left[L_{w}: \mathbb{Q} w\right]}{[L: \mathbb{Q}]} \log \left(C_{w}\right) \\
& \left.+\min \left\{\max _{1 \leq j \leq \operatorname{dim} F} h\left(s_{j}\right) \mid\left(s_{1}, \ldots, s_{\operatorname{dim} F}\right) \text { base de } F\right\}\right) .
\end{aligned}
$$

En particulier, ces points sont en nombre fini si $\mathscr{L}$ est ample.

On donne maintenant un énoncé avec des bases fixées. Soient $w \in \mathscr{P}$ et $k_{w} \in\left\{0, \ldots, e_{w}\right\}$ un entier. Pour tout entier $k$ tel que $0 \leq k \leq e_{w}$, on fixe une base $\left(s_{w, k, l}\right)_{l}$ de $V_{k}^{(w)}$.

Corollaire 4.4. Supposons que l'espace vectoriel

$$
F=\bigcap_{w \in \mathscr{P}} V_{k_{w}}^{(w)}=\bigcap_{w \in \mathscr{P}} \mathscr{F}_{w}^{c_{w}, k_{w}} V_{L}
$$

soit non nul. Si $\delta:=\sum_{w \in \mathscr{P}} \frac{\left[L_{w}: \mathbb{Q}_{w}\right]}{[L: \mathbb{Q}]} c_{w, k_{w}}>1$, alors tous les points $x \in(X \backslash$ $\mathscr{B}(F))(K)$ satisfaisant le système d'inégalités

$$
\left\|s_{w, k_{w}, l}(x)\right\|_{w} \leq \exp \left(-c_{w, k_{w}} h_{\mathscr{L}}(x)\right), \quad \forall 1 \leq l \leq \operatorname{dim}\left(V_{k_{w}}^{(w)}\right), \quad \forall w \in \mathscr{P}
$$

vérifient

$$
h_{\mathscr{L}}(x) \leq \frac{1}{\delta-1}\left(-\sum_{w \in \mathscr{P}} \frac{\left[L_{w}: \mathbb{Q} w\right]}{[L: \mathbb{Q}]} \log \left(\tau_{w}\right)+\max _{1 \leq j \leq \operatorname{dim} F} h\left(s_{j}\right)\right),
$$

où $\left(s_{1}, \ldots, s_{\operatorname{dim} F}\right)$ est une base quelconque de $F$ et les $\tau_{w}$ sont des nombres réels non nuls ne dépendant que de la base $\left(s_{w, k_{w}, l}\right)_{l}$ et du choix de métrique sur $\mathscr{L}$. Dans le cas particulier où $\mathscr{P}=\{w\}$ n'a qu'un élément, on a de plus

$$
h_{\mathscr{L}}(x) \leq \frac{1}{\delta-1}\left(-\frac{\left[L_{w}: \mathbb{Q} w\right]}{[L: \mathbb{Q}]} \min _{l} \log \left(\left\|s_{w, k_{w}, l}\right\|_{w, \text { sup }}\right)+\max _{l} h\left(s_{w, k_{w}, l}\right)\right) .
$$

Démonstration : Soit $x \in(X \backslash \mathscr{B}(F))(K)$ vérifiant le système d'inégalités du corollaire. Soit $w \in \mathscr{P}$. Si $w$ est une place finie, soit $\left.\left.\alpha_{w} \in\right] 0,1\right]$ la borne supérieure des nombres réels $\alpha \in] 0,1\left[\right.$ tels que la base $\left(s_{w, k_{w}, l}\right)_{l}$ soit $\alpha$-orthogonale pour $w$, c'est à dire

$$
\left\|\sum_{l} a_{l} s_{w, k_{w}, l}\right\|_{w, \text { sup }} \geq \alpha \max _{l}\left(\left|a_{l}\right|_{w}\left\|s_{w, k_{w}, l}\right\|_{w, \text { sup }}\right)
$$

pour tout $\sum_{l} a_{l} s_{w, k_{w}, l} \in V_{L}$. Si $w$ est archimédienne, alors par équivalence des normes il existe un nombre réel $\alpha_{w}$ tel que

$$
\left\|\sum_{l} a_{l} s_{w, k_{w}, l}\right\|_{w, \text { sup }} \geq \alpha_{w} \sum_{l}\left(\left|a_{l}\right|_{w}\left\|s_{w, k_{w}, l}\right\|_{w, \text { sup }}\right)
$$


pour tout $\sum_{l} a_{l} s_{w, k_{w}, l} \in V_{L}$. On a ainsi

$$
\begin{aligned}
\left\|\sum_{l} a_{l} s_{w, k_{w}, l}\right\|_{w, \text { sup }} & \geq \alpha_{w} \sum_{l}\left(\left|a_{l}\right|_{w}\left\|s_{w, k_{w}, l}\right\|_{w, \text { sup }}\right) \\
& \geq \alpha_{w}\left(\sum_{l}\left|a_{l}\right|_{w}\right) \cdot \min _{l}\left\|s_{w, k_{w}, l}\right\|_{w, \text { sup }} .
\end{aligned}
$$

Considérons une section non nulle $s=\sum_{l} a_{l} s_{w, k_{w}, l}$ dans $V_{k_{w}}^{(w)}$; si $w$ est finie, on a alors

$$
\begin{aligned}
\|s(x)\|_{w}=\left\|\sum_{l} a_{l} s_{w, k_{w}, l}(x)\right\|_{w} & \leq \max _{l}\left|a_{l}\right|_{w} \cdot\left\|s_{w, k_{w}, l}(x)\right\|_{w} \\
& \leq \exp \left(-c_{w, k_{w}} h_{\mathscr{L}}(x)\right) \max _{l}\left|a_{l}\right|_{w} .
\end{aligned}
$$

On démontre de la même façon un résultat analogue si $w \mid \infty$. En notant $\tau_{w}=$ $\alpha_{w} \min _{l}\left\|s_{w, k_{w}, l}\right\|_{w, \text { sup }}$, le point $x$ est aussi solution du système

$$
\frac{\|s(x)\|_{w}}{\|s\|_{w, \text { sup }}} \leq \tau_{w}^{-1} \exp \left(-c_{w, k_{w}} h_{\mathscr{L}}(x)\right), \quad \forall s \in V_{k_{w}}^{(w)} \backslash\{0\}, \forall k_{w}, \forall w \in \mathscr{P} .
$$

En appliquant le corollaire 4.2, on obtient que

$$
h_{\mathscr{L}}(x) \leq \frac{1}{\delta-1}\left(-\sum_{w \in \mathscr{P}} \frac{\left[L_{w}: \mathbb{Q} w\right]}{[L: \mathbb{Q}]} \log \left(\tau_{w}\right)+\max _{1 \leq j \leq \operatorname{dim} F} h\left(s_{j}\right)\right),
$$

où $\left(s_{1}, \ldots, s_{\operatorname{dim} F}\right)$ est une base quelconque de $F$. Si $\mathscr{P}=\{w\}$ n'a qu'un élément, il suffit d'appliquer le théorème 4.1 avec $C_{w}=\left(\min _{l}\left\|s_{w, k_{w}, l}\right\|_{w, \text { sup }}\right)^{-1}$.

Dans le cas où l'ensemble $\mathscr{P}=\{w\}$ n'a qu'un élément, ce résultat implique le théorème 1.1 de Faltings et Wüstholz ([6, théorème 9.1]). En effet, si on note $W$ le sous-espace déstabilisant de la filtration $\mathscr{F}_{w}$, on a dans ce cas $W=V_{e_{w}}^{(w)}$ et $\mu_{w}(W)=c_{w, e_{w}}$. Plus généralement, si $F:=\cap_{w} V_{w, e_{w}} \neq\{0\}$, le corollaire 4.4 redonne le théorème 1.1 avec $Z=\mathscr{B}(F) \subsetneq X$ (en utilisant le fait que $c_{w, e_{w}} \geq \mu_{w}(W)$ pour toute place $\left.w \in \mathscr{P}\right)$. Cependant dans le cas général le corollaire 4.4 n'implique pas le théorème 1.1 car l'ensemble $\mathscr{B}(F)$ peut être égal à $X$ tout entier. L'intérêt des résultats de cette partie, en plus de fournir une majoration explicite de la hauteur des solutions, est qu'ils donnent un lieu de base facile à déterminer en dehors duquel se situent les points satisfaisant le système d'inégalités. Ces nouveautés sont cruciales pour les applications que nous avons en vue dans la partie 5 . Nous utiliserons les résultats de cette partie dans le cas particulier où $\mathscr{P}$ est un singleton.

\section{Le théorème de Liouville effectif : seconde ap- proche}

Dans cette partie, nous démontrons le théorème 1.4 de l'introduction (dont le corollaire 1.5 est une conséquence). Dans l'article [13], McKinnon et Roth utilisent 
le théorème 9.1 de l'article [6] pour établir une inégalité entre une constante d'approximation et la constante de Seshadri et démontrent ainsi une généralisation du théorème de Roth. Nous reprenons ici ces idées en utilisant à la place le théorème 4.1. Les constantes qui interviendront dans cette partie ont l'avantage de ne pas dépendre d'un choix de métriques sur le diviseur exceptionnel, à la différence de celles de la partie 3.

\subsection{Cas général}

Soit $K$ un corps de nombres et $X$ une variété projective de dimension $d \geq 1$ sur $\operatorname{Spec}(K)$. On considère un fibré inversible adélique $\overline{\mathscr{L}}=\left(\mathscr{L},\left(\|\cdot\|_{v}\right)_{v \in \Sigma_{K}}\right)$ sur $X$ avec $\mathscr{L}$ nef et un point régulier $x \in X(\bar{K})$. On note $L$ le corps de définition de $x, \mathscr{O}_{X, x}$ l'anneau local défini par $x, \mathfrak{M}_{x}$ son idéal maximal, $\pi: \widetilde{X} \rightarrow$ $X_{L}$ l'éclatement de $X_{L}=X \times_{K} \operatorname{Spec}(L)$ en $x$ et $E$ le diviseur exceptionnel associé. Comme dans la partie 3 , nous noterons $\mathscr{L}_{\gamma}=\pi^{*} \mathscr{L}-\gamma E$ pour tout rationnel positif $\gamma$. Dans leurs articles [13] et [14], McKinnon et Roth utilisent des méthodes asymptotiques pour démontrer des analogues des théorèmes de Roth et de Liouville en dimension quelconque en considérant des suites de points qui convergent vers $x$ relativement à une distance. Pour cela ils comparent le comportement asymptotique des générateurs de $\mathfrak{M}_{x}$ et de la fonction distance (voir la partie 2 de [13]). A cet effet ils n'ont pas besoin d'expliciter les constantes qui interviennent. Le lemme 5.2 est le résultat clé qui nous permettra d'obtenir des constantes explicites, puis une version effective du théorème de Liouville. On peut l'interpréter comme un analogue du lemme de Schwarz valable pour les sections globales de $\mathscr{L}$. Dans la suite, on fixe une place $v$ de $L$ et un plongement $\varphi: X \hookrightarrow \mathbb{P}_{K}^{n}$ et on considère la distance $d_{v}$ associée à ce plongement. Nous commençons par démontrer un résultat d'approximation des fonctions distances.

Lemme 5.1. Si $v$ est archimédienne, alors pour tout $x^{\prime} \in \mathbb{P}^{n}\left(\mathbb{C}_{v}\right)$ il existe un plongement $\varphi^{\prime}: X \hookrightarrow \mathbb{P}_{\mathbb{C}_{v}}^{n}$ envoyant $x$ sur $x^{\prime}$ et définissant une distance $d_{v}^{\prime}$ vérifiant : $d_{v}^{\prime}(x, y)=d_{v}(x, y)$ pour tout $y \in X\left(\mathbb{C}_{v}\right)$.

Si $v$ est ultramétrique, alors pour tout $x^{\prime} \in \mathbb{P}^{n}\left(\mathbb{C}_{v}\right)$ et pour tout $\varepsilon>0$, il existe un plongement $\varphi_{\varepsilon}^{\prime}: X \hookrightarrow \mathbb{P}_{\mathbb{C}_{v}}^{n}$ envoyant $x$ sur $x^{\prime}$ et définissant une distance $d_{v}^{\prime}$ vérifiant

$$
(1+\varepsilon)^{-1} d_{v}(x, y) \leq d_{v}^{\prime}(x, y) \leq(1+\varepsilon) d_{v}(x, y)
$$

pour tout $y \in X\left(\mathbb{C}_{v}\right)$.

Démonstration : Supposons que $v$ est archimédienne; alors $\varphi(x)$ appartient à $\mathbb{P}^{n}(\mathbb{C})$. On choisit un représentant $\widetilde{x}$ de $\varphi(x)$ dans $\mathbb{C}^{n+1} \backslash\{0\}$ tel que $|\widetilde{x}|_{2, v}=1$, où la norme $|\cdot|_{2, v}$ est définie par $\left|\left(z_{0}, \ldots, z_{n}\right)\right|_{2, v}=\left(\sum_{i}\left|z_{i}\right|_{v}^{2}\right)^{1 / 2}$, associée au choix de coordonnées défini par $\varphi$ ). On construit une base orthonormée $\mathrm{u}=$ $\left(x, u_{1}, \ldots, u_{n}\right)$ de $\mathbb{C}^{n+1}$ par le procédé d'orthonormalisation de Gram-Schmidt. Soit $x^{\prime} \in \mathbb{C}^{n+1} \backslash\{0\}$ tel que $\left|x^{\prime}\right|_{2, v}=1$. De la même façon on construit une base orthonormée $\mathrm{v}=\left(x^{\prime}, v_{1}, \ldots, v_{n}\right)$. Il existe un unique automorphisme unitaire $T$ de $\mathbb{C}^{n+1}$ envoyant la base u sur v. Cet automorphisme induit un changement de coordonnées $\phi$ sur $\mathbb{P}_{\mathbb{C}}^{n}$ tel que $\phi(x)=x^{\prime}$. On note $\varphi^{\prime}=\phi \circ \varphi$ et $d_{v}^{\prime}$ la distance associée. On a bien $d_{v}(x, y)=d_{v}^{\prime}(x, y)$ pour tout $y \in X\left(K_{v}\right)$ puisque $T$ est unitaire.

Supposons maintenant que $v$ est ultramétrique. Soit $\varepsilon>0$ et $\varepsilon^{\prime}$ tel que $(1+$ $\varepsilon)^{-1} \leq\left(1-\varepsilon^{\prime}\right)^{2}$. Soit $\widetilde{x}$ un représentant de $\varphi(x)$ dans $\mathbb{C}_{v}^{n+1} \backslash\{0\}$ tel que $|\widetilde{x}|_{2, v}=1$, 
$x^{\prime} \in \mathbb{C}^{n+1} \backslash\{0\}$ tel que $\left|x^{\prime}\right|_{2, v}=1$, et soit $\left.\varepsilon \in\right] 0,1[$. D'après la proposition 3.1 de [2], on peut construire des bases $\left(\widetilde{x}, u_{1}, \ldots, u_{n}\right)$ et $\left(x^{\prime}, u_{1}^{\prime}, \ldots, u_{n}^{\prime}\right)$ de $\mathbb{C}_{v}^{n+1}$ avec $\left|u_{i}\right|_{2, v}=\left|u_{i}^{\prime}\right|_{2, v}=1$ pour tout $1 \leq i \leq n$ et telles que pour tout $\left(\lambda_{0}, \ldots, \lambda_{n}\right) \in \mathbb{C}_{v}^{n}$

$$
\left|\lambda_{0} \widetilde{x}+\sum_{i=1}^{n} \lambda_{i} u_{i}\right|_{2, v} \geq\left(1-\varepsilon^{\prime}\right) \max _{0 \leq i \leq n}\left|\lambda_{i}\right|_{v}
$$

et

$$
\left|\lambda_{0} x^{\prime}+\sum_{i=1}^{n} \lambda_{i} u_{i}^{\prime}\right|_{2, v} \geq\left(1-\varepsilon^{\prime}\right) \max _{0 \leq i \leq n}\left|\lambda_{i}\right|_{v}
$$

Soit $T_{\varepsilon}$ l'automorphisme de $\mathbb{C}_{v}^{n+1}$ tel que $T_{\varepsilon}(\widetilde{x})=x^{\prime}$ et $T_{\varepsilon}\left(u_{i}\right)=u_{i}^{\prime}$ pour tout $1 \leq i \leq n$. On note $\varphi_{\varepsilon}^{\prime}=T_{\varepsilon} \circ \varphi: X \rightarrow \mathbb{P}_{\mathbb{C}_{v}}^{n}$ et $d_{v}^{\prime}$ la distance associée. D'après le théorème 3 de [4], on a l'inégalité

$$
\eta_{v}\left(T_{\varepsilon}\right)^{-1} d_{v}(x, y) \leq d_{v}^{\prime}(x, y) \leq \eta_{v}\left(T_{\varepsilon}\right) d_{v}(x, y),
$$

où $\eta_{v}\left(T_{\varepsilon}\right)=\left\|T_{\varepsilon}\right\|_{v}\left\|T_{\varepsilon}^{-1}\right\|_{v}$. Ici $\left\|T_{\varepsilon}\right\|_{v}$ désigne la norme matricielle associée à la norme $|\cdot|_{2, v}:\left\|T_{\varepsilon}\right\|_{v}=\sup \left\{\left|T_{\varepsilon}(u)\right|_{2, v} /|u|_{2, v} \mid u \in \mathbb{C}_{v}^{n+1} \backslash\{0\}\right\}$. On a les majorations

$$
\left\|T_{\varepsilon}\right\|_{v} \leq \frac{1}{1-\varepsilon^{\prime}} \text { et }\left\|T_{\varepsilon}^{-1}\right\|_{v} \leq \frac{1}{1-\varepsilon^{\prime}},
$$

d'où

$$
\frac{d_{v}(x, y)}{(1+\varepsilon)} \leq\left(1-\varepsilon^{\prime}\right)^{2} d_{v}(x, y) \leq d_{v}^{\prime}(x, y) \leq \frac{d_{v}(x, y)}{\left(1-\varepsilon^{\prime}\right)^{2}} \leq(1+\varepsilon) d_{v}(x, y) .
$$

Afin de comparer les normes de sections de $\mathscr{L}$ avec la fonction distance, nous allons utiliser l'inégalité de Cauchy dans le cas complexe et son analogue dans le cas ultramétrique. Pour cela nous devrons travailler sur des disques de $\mathbb{C}_{v}^{d}$. Pour tout nombre réel $\rho>0$, on considère la boule

$$
B(\rho, \varphi)=\left\{z \in X\left(\mathbb{C}_{v}\right) \mid d_{v}(x, z)<\rho\right\} .
$$

Rappelons que le point $x \in X(\bar{K})$ est supposé régulier. D'après le corollaire 6.2.11 de [12] (voir aussi la proposition 3.24 de [15]), il existe un ouvert $U$ de $X_{L}$ contenant $x$ et sur lequel $\mathscr{L}$ est trivial ainsi qu'un morphisme étale $g: U \rightarrow \mathbb{A}_{L}^{d}$. Il existe alors un polydisque $D$ dans $\mathbb{C}_{v}^{d}$ et un ouvert $V_{D}$ de $U\left(\mathbb{C}_{v}\right)$ (pour la topologie $v$-adique) tels que $g$ induise un isomorphisme entre $V_{D}$ et $D$. On définit alors

$$
\rho_{x}\left(\mathscr{L}, \varphi, U, V_{D}\right)=\sup \left\{\rho>0 \mid B(\rho, \varphi) \subset V_{D}\right\} .
$$

Enfin, nous définissons $\rho_{x}(\mathscr{L}, \varphi)$ comme la borne supérieure des $\rho_{x}\left(\mathscr{L}, \varphi, U, V_{D}\right)$ pour $U$ et $V_{D}$ parcourant les ouverts satisfaisant les conditions précédentes. Cette quantité dépend du plongement $\varphi: X \hookrightarrow \mathbb{P}^{n}$ fixé. Dans la suite, s'il n'y a pas d'ambiguïté nous noterons plus simplement $\rho_{x}(\mathscr{L})=\rho_{x}(\mathscr{L}, \varphi)$ et $B(\rho)=$ $B(\rho, \varphi)$. Remarquons que dans le cas où $X=\mathbb{P}^{n}$ et $\mathscr{L}=\mathscr{O}(\ell)$ avec $\ell \in \mathbb{N} \backslash\{0\}$, alors $\rho_{x}(\mathscr{L}) \geq 1$. En effet, si $d_{v}(x, y)<1$ alors il existe $i_{0}$ tel que $x_{i_{0}} y_{i_{0}} \neq 0$ (pour un choix convenable de coordonnées homogènes) et donc $B(1)$ est inclus dans un ouvert satisfaisant les conditions précédentes. 
Dans la suite, nous noterons $a(v)=1$ si $v$ est archimédienne et $a(v)=0$ sinon. L'énoncé suivant est au coeur de la démonstration du théorème 5.3. Il permet de comparer la norme de l'évaluation d'une section en un point $y \in X(K)$ avec la distance $d_{v}(x, y)$. Cette technique est utilisée par McKinnon et Roth sous une forme qui n'est pas effective dans l'article [13] (notamment dans la démonstration de leur théorème 5.1) pour démontrer un analogue du théorème de Roth. À notre connaissance, il n'existe pas de résultat similaire explicite dans la littérature à l'heure actuelle.

Lemme 5.2. Soient $r$ un entier naturel et $s \in \mathrm{H}^{0}(X, \mathscr{L}) \backslash\{0\}$ une section s'annulant à l'ordre $r$ en $x$. Alors pour tout point $y \in X\left(\mathbb{C}_{v}\right)$ distinct de $x$ tel que $d_{v}(x, y)<1 / 2^{a(v)}$, l'inégalité suivante est satisfaite :

$$
\frac{\|s(y)\|_{v}}{\|s\|_{v, \text { sup }}} \leq\left(2^{a(v)} \frac{d_{v}(x, y)}{\rho_{x}(\mathscr{L})}\right)^{r} .
$$

Démonstration: On note $T_{0}, \ldots, T_{n}$ les coordonnées homogènes sur $\mathbb{P}^{n}$ correspondant à la distance $d_{v}$. Supposons dans un premier temps que $\varphi$ envoie le point $x \operatorname{sur}(1: 0: \cdots: 0) \in \mathbb{P}^{n}$. Considérons l'ouvert $U_{0}$ de $\mathbb{P}^{n}$ défini par $T_{0} \neq 0$. Pour $1 \leq i \leq n$, on note $u_{i}=T_{i} / T_{0} \in \mathscr{O}_{\mathbb{P} n}\left(U_{0}\right)$. On note encore $u_{i}$ la restriction à $\varphi^{-1}\left(U_{0}\right)$. D'après la preuve du lemme 2.5 de [13],

$$
\min \left\{1, \max \left(\left|u_{1}(z)\right|_{v}, \ldots,\left|u_{n}(z)\right|_{v}\right)\right\} \leq 2^{a(v)} d_{v}(x, z)
$$

pour tout $z \in \varphi^{-1}\left(U_{0}\right)\left(\mathbb{C}_{v}\right)$. Soit $y \in X\left(\mathbb{C}_{v}\right)$ distinct de $x$ tel que $d_{v}(x, y)<$ $1 / 2^{a(v)}$ et $s \in \mathrm{H}^{0}\left(X, \mathscr{L} \otimes \mathfrak{M}_{x}^{r}\right) \backslash\{0\}$. Si $d_{v}(x, y) \geq \rho_{x}(\mathscr{L})$ alors le résultat découle simplement de la majoration

$$
\frac{\|s(y)\|_{v}}{\|s\|_{v, \text { sup }}} \leq 1 .
$$

Dans la suite, on suppose donc $d_{v}(x, y)<\rho_{x}(\mathscr{L})$. Le point $y$ appartient à $\varphi^{-1}\left(U_{0}\right)$ (car sinon $\left.d_{v}(x, y)=1\right)$ et donc

$$
\max \left(\left|u_{1}(y)\right|_{v}, \ldots,\left|u_{n}(y)\right|_{v}\right) \leq 2^{a(v)} d_{v}(x, y) .
$$

Soit $\rho$ un nombre réel tel que $d_{v}(x, y)<\rho<\rho_{x}(\mathscr{L})$. Rappelons qu'il existe un morphisme étale $g: B(\rho, \varphi) \subset U \rightarrow \mathbb{A}_{L}^{d}$ où $U$ est un ouvert de Zariski sur lequel $\mathscr{L}$ est trivial : $\mathscr{L}_{U}=s_{0} \cdot \mathscr{O}_{U}$. Quitte à renuméroter, on peut supposer que $u_{1}, \ldots, u_{d}$ engendrent l'idéal $\mathfrak{M}_{x}$ (rappelons que $\left.d=\operatorname{dim} X\right)$. Il existe donc un système de paramètres $\left(\widetilde{u}_{1}, \ldots, \widetilde{u}_{d}\right)$ en $g(x)$ tel que $\widetilde{u}_{i}(g(z))=u_{i}(z)$ au voisinage de $x$. Nous allons montrer qu'il existe une trivialisation $\mathscr{L}_{\mid U}=s_{\varepsilon} \cdot \mathscr{O}_{U}$ vérifiant

$$
1-\varepsilon \leq\left\|s_{\varepsilon}(z)\right\|_{v} \leq 1+\varepsilon
$$

pour tout $z \in B(\rho, \varphi)$. Pour tout $z \in B(\rho, \varphi)$, soit un élément $b_{v}(z) \in \mathbb{C}_{v}$ tel que $\left|b_{v}(z)\right|_{v}=\left\|s_{0}(z)\right\|_{v}^{-1}$. On considère l'application continue

$$
\begin{aligned}
\varphi_{v}: \quad B(\rho, \varphi) & \rightarrow \mathbb{C}_{v} \\
z & \mapsto b_{v}(z) .
\end{aligned}
$$

Si $v$ est ultramétrique, il existe un polynôme $P$ tel que pour tout $z \in B(\rho, \varphi)$, $\left|P(z)-\varphi_{v}(z)\right|_{v}<\varepsilon /\left\|s_{0}\right\|_{v \text {,sup }}$ d'après le théorème d'interpolation de [3]. Considérons la section $s_{\varepsilon}=s_{0} \cdot P$. Alors pour tout $z \in B(\rho, \varphi)$,

$$
\left\|s_{\varepsilon}(z)\right\|_{v}=\left\|s_{0}(z)\right\|_{v}|P(z)|_{v} \leq\left\|s_{0}(z)\right\|_{v}\left|\varphi_{v}(z)\right|_{v}+\varepsilon=1+\varepsilon .
$$


De même $\left\|s_{\varepsilon}(z)\right\|_{v} \geq 1-\varepsilon$. Ainsi, pour tout $z \in B(\rho, \varphi)$, on a

$$
1-\varepsilon \leq\left\|s_{\epsilon}(z)\right\|_{v} \leq 1+\varepsilon \text {. }
$$

Dans le cas archimédien, on procède de la même manière en appliquant le théorème de Stone-Weierstrass à $\varphi_{v}: B(\rho, \varphi) \rightarrow \mathbb{R}, z \mapsto\left\|s_{0}(z)\right\|_{v}^{-1}$.

Il existe donc une fonction $f \in \mathscr{O}_{X}(U)$ telle que $s=s_{\varepsilon} \cdot f$. Il existe également un polydisque $D$ dans $\mathbb{C}_{v}^{d}$ et un ouvert $V_{D}$ dans $U\left(\mathbb{C}_{v}\right)$ tels que $g$ induise un isomorphisme entre $V_{D}$ et $D$. La fonction $f$ restreinte à $V_{D}$ s'identifie à une fonction analytique $\widetilde{f}$ sur le polydisque $D$. Par hypothèse, si $d_{v}(x, z)<\rho$ alors $g(z) \in D$, et de plus $\left|\widetilde{u}_{i}(g(z))\right|_{v}<\rho$. Donc $\widetilde{f}$ est analytique sur le polydisque $D^{\prime}=\left\{\left.z \in \mathbb{C}_{v}^{d}\left|\max _{1 \leq i \leq d}\right| \widetilde{u}_{i}(z)\right|_{v}<\rho\right\}$ via $g$. Supposons d'abord que $v$ est archimédienne. On note $M=\sup \left\{|\widetilde{f}(z)| \mid z \in D^{\prime}\right\}$. D'après le théorème 3.4 de [10], on en déduit que

$$
\begin{aligned}
\|s(y)\|_{v} \leq(1+\varepsilon)|\widetilde{f}(g(y))| & \leq(1+\varepsilon)\left(\frac{\max _{1 \leq i \leq d}\left|\widetilde{u}_{i}(g(y))\right|}{\rho}\right)^{r} M \\
& \leq(1+\varepsilon)\left(2 \frac{d_{v}(x, y)}{\rho}\right)^{r} M .
\end{aligned}
$$

Par ailleurs, $\|s\|_{v \text {,sup }} \geq(1-\varepsilon) M$. En faisant tendre $\varepsilon$ vers 0 et $\rho$ vers $\rho_{x}(\mathscr{L}, \varphi)$, on obtient bien

$$
\frac{\|s(y)\|_{v}}{\|s\|_{v, \text { sup }}} \leq\left(2 \frac{d_{v}(x, y)}{\rho_{x}(\mathscr{L}, \varphi)}\right)^{r} .
$$

Considérons maintenant le cas où $v$ est ultramétrique. La fonction $f$ s'identifie à une fonction analytique $\widetilde{f}$ sur le polydisque $D^{\prime}$ qui s'annule à un ordre supérieur à $r$ en $g(x)$ et s'écrit

$$
\widetilde{f}=\sum_{r \leq k_{1}+\cdots+k_{n}} a_{k} \widetilde{u}_{1}^{k_{1}} \cdots \widetilde{u}_{n}^{k_{n}}
$$

On a $f(y)=\widetilde{f}(g(y))$ et on obtient

$$
\begin{aligned}
\|s(y)\|_{v}=\left\|s_{\varepsilon}(y)\right\|_{v}|f(y)|_{v} & \leq(1+\varepsilon) \max _{k}\left\{\left|a_{k}\right|_{v} \prod_{1 \leq i \leq n}\left|\widetilde{u}_{i}(g(y))\right|_{v}^{k_{i}}\right\} \\
& \leq(1+\varepsilon) \max _{k}\left(\left|a_{k}\right|_{v} \rho^{|k|-r}\right)\left(d_{v}(x, y)\right)^{r}
\end{aligned}
$$

D'après le lemme 15.1 de [19] appliqué sur le polydisque $D^{\prime}$, on a de plus

$$
\begin{aligned}
\|s\|_{v, \text { sup }} & \geq \sup \left\{\left\|s_{0}(\nu)\right\|_{v}|f(\nu)|_{v} \mid \nu \in B\left(\rho_{x}(\mathscr{L}), \varphi\right)\right\} \\
& \geq(1-\varepsilon) \sup \left\{|\widetilde{f}(\nu)|_{v} \mid \nu \in D^{\prime}\right\} \\
& \geq(1-\varepsilon) \max _{k}\left(\left|a_{k}\right|_{v} \rho^{|k|}\right) .
\end{aligned}
$$

En faisant tendre $\varepsilon$ vers 0 et $\rho$ vers $\rho_{x}(\mathscr{L}, \varphi)$, on obtient

$$
\frac{\|s(y)\|_{v}}{\|s\|_{v, \text { sup }}} \leq\left(\frac{d_{v}(x, y)}{\rho_{x}(\mathscr{L}, \varphi)}\right)^{r} .
$$

Le lemme est donc démontré dans le cas où $\varphi(x)=(1: 0: \cdots: 0)$. Le cas d'un plongement $\varphi$ quelconque se déduit du lemme 5.1 de la façon suivante. Si $v$ est 
ultramétrique, soit $\varepsilon^{\prime}>0$. D'après le lemme 5.1, il existe un plongement $\varphi^{\prime}$ tel que $\varphi^{\prime}(x)=(1: 0: \cdots: 0)$, définissant une distance $d_{v}^{\prime}$ qui vérifie

$$
\left(1+\varepsilon^{\prime}\right)^{-1} d_{v}(x, z) \leq d_{v}^{\prime}(x, z) \leq\left(1+\varepsilon^{\prime}\right) d_{v}(x, z)
$$

pour tout $z \in X\left(\mathbb{C}_{v}\right)$. On en déduit que

$$
\rho_{x}\left(\mathscr{L}, \varphi^{\prime}\right)=\left(1+\varepsilon^{\prime}\right)^{-1} \rho_{x}(\mathscr{L}, \varphi) .
$$

Soit $y \in X\left(\mathbb{C}_{v}\right)$ distinct de $x$ tel que $d_{v}(x, y)<\min \left(1, \rho_{x}(\mathscr{L}, \varphi)\right)$. On suppose $\varepsilon^{\prime}$ suffisamment petit pour avoir $d_{v}^{\prime}(x, y)<\min \left(1, \rho_{x}\left(\mathscr{L}, \varphi^{\prime}\right)\right)$. D'après le cas précédent,

$$
\frac{\|s(y)\|_{v}}{\|s\|_{v, \text { sup }}} \leq\left(\frac{d_{v}^{\prime}(x, y)}{\rho_{x}\left(\mathscr{L}, \varphi^{\prime}\right)}\right)^{r}
$$

donc

$$
\frac{\|s(y)\|_{v}}{\|s\|_{v, \text { sup }}} \leq\left(\left(1+\epsilon^{\prime}\right)^{2} \frac{d_{v}(x, y)}{\rho_{x}(\mathscr{L}, \varphi)}\right)^{r}
$$

On en déduit le résultat voulu en faisant tendre $\varepsilon^{\prime}$ vers 0 . Si $v$ est archimédienne, on conclut également avec le lemme 5.1.

Le résultat principal de cette partie est le théorème suivant, qui est une conséquence du corollaire 4.2 et du lemme 5.2. Rappelons que l'on note $a(v)=1$ si $v$ est archimédienne et $a(v)=0$ sinon.

Théorème 5.3. Soient $\gamma>0$ un nombre rationnel et $r$ un entier non nul tel que $r \gamma \in \mathbb{N}$. On note $F_{r}$ le sous-espace vectoriel de $H^{0}\left(X_{L}, r \mathscr{L}\right)$ des sections qui s'annulent à l'ordre $r \gamma$ en $x$. Alors pour tout $\varepsilon>0$ et pour tout point $y \in\left(X \backslash \mathscr{B}\left(F_{r}\right)\right)(K)$ distinct de $x$ satisfaisant l'inégalité

$$
\log \left(d_{v}(x, y)\right)<-\left(\frac{[L: \mathbb{Q}]}{\gamma\left[L_{v}: \mathbb{Q}_{v}\right]}+\varepsilon\right) h_{\mathscr{L}}(y)
$$

la hauteur $h_{\mathscr{L}}(y)$ est majorée par

$$
\max \left\{\left(\frac{[L: \mathbb{Q}]}{\gamma\left[L_{v}: \mathbb{Q}_{v}\right]}+\varepsilon\right)^{-1} a(v) \log 2, \frac{1}{\varepsilon}\left(c_{1}(\mathscr{L}, x)+c_{2}(\mathscr{L}, x, \gamma, r)\right)\right\}
$$

où

$$
c_{1}(\mathscr{L}, x)=a(v) \log 2-\log \left(\rho_{x}(\mathscr{L})\right)
$$

et

$$
c_{2}(\mathscr{L}, x, \gamma, r)=\frac{[L: \mathbb{Q}]}{r \gamma\left[L_{v}: \mathbb{Q}_{v}\right]} \min \left\{\max _{1 \leq i \leq \ell} h\left(s_{i}\right) \mid\left(s_{i}\right)_{1 \leq i \leq \ell} \text { base de } F_{r}\right\} .
$$

En particulier, les points vérifiant (6) sont en nombre fini si $\mathscr{L}$ est ample.

Démonstration : On note $n_{v}=\frac{[L: \mathbb{Q}]}{\left[L_{v}: \mathbb{Q} v\right]}$. Soient $\varepsilon$ un nombre réel strictement positif et $y \in\left(X \backslash \mathscr{B}\left(F_{r}\right)\right)(K)$ un point distinct de $x$ tel que

$$
\log \left(d_{v}(x, y)\right)<-\left(n_{v} / \gamma+\varepsilon\right) h_{\mathscr{L}}(y)
$$


Soit $s \in F_{r} \backslash\{0\}$; cette section s'annule à l'ordre $r \gamma$ en $x$. On peut supposer que $h_{\mathscr{L}}(y)>\left(\frac{n_{v}}{\gamma}+\varepsilon\right)^{-1} a(v) \log 2$; ainsi l'hypothèse du lemme 5.2 est satisfaite et on obtient

$$
\frac{\|s(y)\|_{v}}{\|s\|_{v, \text { sup }}} \leq\left(2^{a(v)} \frac{d_{v}(x, y)}{\rho_{x}(\mathscr{L})}\right)^{r \gamma} .
$$

On a alors

$$
\begin{aligned}
\frac{\|s(y)\|_{v}}{\|s\|_{v, \text { sup }}} & \leq\left(2^{a(v)} \frac{d_{v}(x, y)}{\rho_{x}(\mathscr{L})}\right)^{r \gamma} \\
& \leq\left(2^{a(v)} \frac{\exp \left(-\left(n_{v} / \gamma+\varepsilon\right) h_{\mathscr{L}}(y)\right)}{\rho_{x}(\mathscr{L})}\right)^{r \gamma} \\
& =\left(2^{a(v)} / \rho_{x}(\mathscr{L})\right)^{r \gamma} \exp \left(-\left(n_{v}+\gamma \varepsilon\right) h_{r \mathscr{L}}(y)\right) .
\end{aligned}
$$

En appliquant le corollaire 4.2 avec $F=F_{r}, C_{v}=\left(2^{a(v)} / \rho_{x}(\mathscr{L})\right)^{r \gamma}$ et $t_{v}=$ $n_{v}+\gamma \varepsilon>n_{v}$, on en déduit que

$$
r h_{\mathscr{L}}(y) \leq h_{r} \mathscr{L}(y) \leq \frac{r}{\varepsilon} \log \left(2^{a(v)} / \rho_{x}(\mathscr{L})\right)+\frac{n_{v}}{\varepsilon \gamma} c^{\prime}(\mathscr{L}, x),
$$

où

$$
\left.c^{\prime}(\mathscr{L}, x)=\min \left\{\max _{1 \leq i \leq r} h\left(s_{i}\right) \mid\left(s_{i}\right)_{1 \leq i \leq r} \text { base de } F_{r}\right)\right\}
$$

Si $\mathscr{L}$ est ample, ces points sont en nombre fini d'après la propriété de Northcott.

Le lemme suivant nous permettra de faire le lien entre le théorème 5.3 et le théorème 3.3 de [14].

Lemme 5.4. 1. Soit $\gamma>0$ un nombre rationnel tel que $\mathscr{L}_{\gamma}$ appartienne au cône effectif de $\operatorname{Pic}_{\mathbb{Q}}(\widetilde{X})$ et soit $r$ un entier tel que $r \gamma \in \mathbb{N} \backslash\{0\}$. Notons $F_{r}$ le sous-espace vectoriel de $\mathrm{H}^{0}\left(X_{L}, r \mathscr{L}\right)$ des sections globales s'annulant à l'ordre $r \gamma$ en $x$. Pour toute section non nulle $\widetilde{s} \in \mathrm{H}^{0}\left(\widetilde{X}, r \mathscr{L}_{\gamma}\right)$, il existe une section $s \in F_{r} \backslash\{0\}$ telle que $\widetilde{s}_{\mid \widetilde{X} \backslash E}=\pi^{*}\left(s_{\mid X \backslash\{x\}}\right)$.

2. On a l'inclusion $\mathscr{B}\left(F_{r}\right) \backslash\{x\} \subset \pi\left(\mathscr{B}\left(r \mathscr{L}_{\gamma}\right)\right)$, où $\mathscr{B}\left(r \mathscr{L}_{\gamma}\right)$ est le lieu de base de $r \mathscr{L}_{\gamma}$.

Démonstration : Le faisceau correspondant à $r \mathscr{L}_{\gamma}$ est

$$
\begin{aligned}
\left(\pi^{*} \mathscr{L}\right)^{\otimes r} \otimes_{\mathscr{O}_{\widetilde{X}}} \mathscr{O}_{\widetilde{X}}(r \gamma) & =\left(\pi^{-1} \mathscr{L}\right)^{\otimes r} \otimes_{\pi^{-1} \mathscr{O}_{X}} \mathscr{O}_{\widetilde{X}} \otimes_{\mathscr{O}_{\widetilde{X}}} \mathscr{O}_{\widetilde{X}}(r \gamma) \\
& =\left(\pi^{-1} \mathscr{L}\right)^{\otimes r} \otimes_{\pi^{-1} \mathscr{O}_{X}} \mathscr{O}_{\widetilde{X}}(r \gamma) .
\end{aligned}
$$

D'après la proposition 8.1.12 (e) de [12], on a $\mathscr{O}_{\widetilde{X}}(1)=I_{x} \cdot \mathscr{O}_{\widetilde{X}}$, où $I_{x}$ est le faisceau d'idéaux de $\mathscr{O}_{X}$ associé à $x$. On en déduit que

$$
r \mathscr{L}_{\gamma}=\left(\pi^{-1} \mathscr{L}\right)^{\otimes r} \otimes_{\pi^{-1} \mathscr{O}_{X}}\left(\pi^{-1} I_{x}\right)^{\otimes r \gamma} \otimes_{\pi^{-1} \mathscr{O}_{X}} \mathscr{O}_{\widetilde{X}}
$$

Si $\operatorname{dim} X=1$ alors le point $x$ correspond à un diviseur de Cartier et l'éclatement $\pi: \widetilde{X} \rightarrow X$ est un isomorphisme et donc $r \mathscr{L}_{\gamma}=\pi^{-1}\left(\mathscr{L}^{\otimes r} \otimes_{\mathscr{O}_{X}} I_{x}^{\otimes r \gamma}\right)$. 
Si $\operatorname{dim} X \geq 2$, l'éclatement $\pi$ induit un isomorphisme entre $\tilde{X} \backslash E$ et $X_{L} \backslash\{x\}$. Soit $\widetilde{s} \in \mathrm{H}^{0}\left(\widetilde{X}, r \mathscr{L}_{\gamma}\right)$ une section non nulle. D'après l'égalité (7), la restriction de $\widetilde{s}$ à $\widetilde{X} \backslash E$ s'identifie à une section (non nulle) $s$ de $r \mathscr{L}$, définie sur $X \backslash\{x\}$. Le point $x$ est régulier, donc est contenu dans un ouvert régulier de Zariski $U$ de $X$. Le sous-schéma ouvert $U$ est intègre et normal (car un anneau local régulier est intègre et normal), donc $\operatorname{dim} U=\operatorname{dim} X \geq 2$ et d'après le théorème 4.1.14 de [12], toute section de $\mathrm{H}^{0}\left(X_{L} \backslash\{x\}, \mathscr{L}\right)$ se prolonge de façon unique en une section globale de $\mathscr{L}$. Ainsi $s$ se prolonge en une section globale de $r \mathscr{L}$; cette section s'annule à l'ordre $r \gamma$ en $x$ d'après l'égalité $(7)$.

Pour montrer l'inclusion $\mathscr{B}\left(F_{r}\right) \backslash\{x\} \subset \pi\left(\mathscr{B}\left(r \mathscr{L}_{\gamma}\right)\right)$, il suffit de remarquer que si $z \neq x$, alors $\pi^{-1}(z) \notin E$ et d'utiliser la première partie du lemme.

Le corollaire suivant est une conséquence immédiate du théorème 5.3 et du lemme 5.4 .

Corollaire 5.5. Soit $\gamma>0$ un nombre rationnel tel que $\mathscr{L}_{\gamma}$ soit dans le cône effectif de $\operatorname{Pic}_{\mathbb{Q}}(\widetilde{X})$. Soit $r \in \mathbb{N} \backslash\{0\}$ tel que $r \gamma$ soit entier. On note $\mathscr{B}\left(r \mathscr{L}_{\gamma}\right)$ le lieu de base de $r \mathscr{L}_{\gamma}$ et $\mathscr{B}=\pi\left(\mathscr{B}\left(r \mathscr{L}_{\gamma}\right)\right)$. On pose

$$
c_{1}(\mathscr{L}, x)=a(v) \log 2-\log \left(\rho_{x}(\mathscr{L})\right)
$$

et

$$
c_{2}(\mathscr{L}, x, \gamma, r)=\frac{[L: \mathbb{Q}]}{r \gamma\left[L_{v}: \mathbb{Q}_{v}\right]} \min \left\{\max _{1 \leq i \leq \ell} h\left(s_{i}\right) \mid\left(s_{i}\right)_{1 \leq i \leq \ell} \text { base de } F_{r}\right\},
$$

où $F_{r}$ est le sous-espace vectoriel de $H^{0}\left(X_{L}, r \mathscr{L}\right)$ des sections qui s'annulent à l'ordre $r \gamma$ en $x$. Soit $\varepsilon>0$.

1. Pour tout point $y \in(X \backslash \mathscr{B})(K)$ distinct de $x$ satisfaisant l'inégalité

$$
\log \left(d_{v}(x, y)\right)<-\left(\frac{[L: \mathbb{Q}]}{\gamma\left[L_{v}: \mathbb{Q}_{v}\right]}+\varepsilon\right) h_{\mathscr{L}}(y),
$$

la hauteur $h_{\mathscr{L}}(y)$ est majorée par

$$
\max \left\{\left(\frac{[L: \mathbb{Q}]}{\gamma\left[L_{v}: \mathbb{Q}_{v}\right]}+\varepsilon\right)^{-1} a(v) \log 2, \frac{1}{\varepsilon}\left(c_{1}(\mathscr{L}, x)+c_{2}(\mathscr{L}, x, \gamma, r)\right)\right\} .
$$

En particulier, ces points sont en nombre fini si $\mathscr{L}$ est ample.

2. Si de plus $x \notin \mathscr{B}$, alors pour tout point $y \in X(K)$ distinct de $x$ satisfaisant l'inégalité (8), la hauteur $h_{\mathscr{L}}(y)$ est majorée par

$$
\begin{array}{r}
\max \left\{\left(\frac{[L: \mathbb{Q}]}{\gamma\left[L_{v}: \mathbb{Q} v\right]}+\varepsilon\right)^{-1} \max \left\{-\log \left(d_{v}(x, \mathscr{B})\right), a(v) \log 2\right\},\right. \\
\left.\frac{1}{\varepsilon}\left(c_{1}(\mathscr{L}, x)+c_{2}(\mathscr{L}, x, \gamma, r)\right)\right\},
\end{array}
$$

où $d_{v}(x, \mathscr{B})=\inf _{z \in \mathscr{B}} d_{v}(x, z)$. En particulier, ces points sont en nombre fini si $\mathscr{L}$ est ample. 
Démonstration : D'après le lemme 5.4, les hypothèses $y \notin \mathscr{B}$ et $y \neq x$ entraînent $y \notin \mathscr{B}\left(F_{r}\right)$. Le premier point est alors une conséquence immédiate du théorème 5.3. Supposons de plus que $x \notin \mathscr{B}$. Soit $y \in X(K)$ un point distinct de $x$ satisfaisant l'inégalité (8). Si $y \notin \mathscr{B}$, le point 2 est une conséquence du point 1. Si $y \in \mathscr{B}$, alors $d_{v}(x, y) \geq d_{v}(x, \mathscr{B})$, donc

$$
h_{\mathscr{L}}(y) \leq-\left(\frac{[L: \mathbb{Q}]}{\gamma\left[L_{v}: \mathbb{Q}_{v}\right]}+\varepsilon\right)^{-1} \log \left(d_{v}(x, \mathscr{B})\right) .
$$

Remarque 5.6. Le corollaire 5.5 implique le théorème 1.4 de la façon suivante. Dans le théorème 1.4 intervient le lieu de base stable $\mathbf{B}\left(\mathscr{L}_{\gamma}\right)$ de $\mathscr{L}_{\gamma} \in \operatorname{Pic}_{\mathbb{Q}}(\widetilde{X})$ à la place de $\mathscr{B}\left(r \mathscr{L}_{\gamma}\right)$. Mais d'après la proposition 2.1.21 de [11], on peut choisir $r$ tel que $\mathbf{B}\left(\mathscr{L}_{\gamma}\right)=\mathscr{B}\left(r \mathscr{L}_{\gamma}\right)$. Il suffit alors d'appliquer le corollaire 5.5 avec ce choix de $r$ pour obtenir le théorème 1.4. En pratique, il peut cependant s'avérer difficile de déterminer un entier $r$ tel que $\mathbf{B}\left(\mathscr{L}_{\gamma}\right)=\mathscr{B}\left(r \mathscr{L}_{\gamma}\right)$. Cette difficulté est atténuée par le fait que le théorème 5.3 et le corollaire 5.5 sont valables pour tout entier $r$ non nul tel que $r \gamma \in \mathbb{N}$, pourvu que l'on ne considère que des points $y \in X(K)$ en dehors de $\mathscr{B}\left(F_{r}\right)$ et $\mathscr{B}\left(r \mathscr{L}_{\gamma}\right)$.

Nous allons maintenant voir que dans le cas où $\mathscr{L}$ est ample et $\gamma<\epsilon_{x}(\mathscr{L})$, il est possible de remplacer $\mathscr{B}$ par $\{x\}$ sans considérer d'entier $r$ arbitrairement grand dans le corollaire 5.5 , à condition que $\mathscr{O}_{\widetilde{X}}(E)$ soit muni de métriques convenables au sens de la définition suivante.

Définition 5.7. Nous dirons qu'une métrique adélique sur $\mathscr{O}_{\widetilde{X}}(E)$ vérifie la condition $(A)$ si pour tout $(p, q) \in(\mathbb{N} \backslash\{0\})^{2}$ tel que $p\left(\pi^{*} \mathscr{L}\right)-q E$ soit ample, la métrique induite sur $p \pi^{*} \mathscr{L}-q E$ est semi-positive et vérifie : pour tout point $z \in(\widetilde{X} \backslash E)\left(\mathbb{C}_{v}\right)$ et pour toute section globale $\widetilde{s}$ de $p\left(\pi^{*} \mathscr{L}\right)-q E$ ne s'annulant pas en $z$, pour toute place $v \in \Sigma_{L}$

$$
\|\widetilde{s}(z)\|_{v}=\|s(\pi(z))\|_{v}
$$

où $s$ est la section de $r \mathscr{L}$ associée à $\widetilde{s}$ du lemme 5.4 .

On obtient le théorème suivant, qui est à mettre en parallèle avec le théorème 3.1 .

Théorème 5.8. Supposons que $\mathscr{L}$ est ample et que le fibré $\mathscr{O}_{\widetilde{X}}(E)$ est muni d'une métrique vérifiant la condition $(A)$. Soit $0<\gamma<\epsilon_{x}(\mathscr{L})$ un nombre rationnel. Alors pour tout $\varepsilon>0$, pour tout point $y \in X(K)$ distinct de $x$ satisfaisant l'inégalité

$$
\log \left(d_{v}(x, y)\right)<-\left(\frac{[L: \mathbb{Q}]}{\gamma\left[L_{v}: \mathbb{Q}_{v}\right]}+\varepsilon\right) h_{\mathscr{L}}(y),
$$

la hauteur $h_{\mathscr{L}}(y)$ est majorée par

$$
\max \left\{\left(\frac{[L: \mathbb{Q}]}{\gamma\left[L_{v}: \mathbb{Q}_{v}\right]}+\varepsilon\right)^{-1} a(v) \log 2, \frac{1}{\varepsilon}\left(c_{1}(\mathscr{L}, x)+c_{3}(\mathscr{L}, x, \gamma)\right)\right\},
$$

où

$$
c_{1}(\mathscr{L}, x)=a(v) \log 2-\log \left(\rho_{x}(\mathscr{L})\right)
$$


et

$$
c_{3}(\mathscr{L}, x, \gamma)=-\frac{[L: \mathbb{Q}]}{\gamma\left[L_{v}: \mathbb{Q}_{v}\right]}\left(\mathrm{e}(\overline{\mathscr{L}})+\gamma \min \left\{0, \inf _{z \in E(\overline{\mathbb{Q}})} h_{\mathscr{O}(-E)}(z)\right\}\right) .
$$

En particulier, ces points sont en nombre fini.

Remarquons que le fibré $\mathscr{O}(-E)=\mathscr{O}_{\widetilde{X}}(1)$ est un fibré ample, donc la quantité $\inf _{z \in E(\overline{\mathbb{Q}})} h_{\mathscr{O}(-E)}(z)$ est bien finie.

Démonstration : Comme $\gamma<\epsilon_{x}(\mathscr{L}), \mathscr{L}_{\gamma}$ est ample. Soit $m$ un entier non nul tel que $m \gamma \in \mathbb{N}$ et soit $r \in \mathbb{N}$ tel que $r m \mathscr{L}_{\gamma}$ soit très ample (en particulier $\left.\left.\mathscr{B}\left(r m \mathscr{L}_{\gamma}\right)\right)=\emptyset\right)$. On note $n_{v}=\frac{[L: \mathbb{Q}]}{\left[L_{v}: \mathbb{Q}_{v}\right]}$. Soient $\varepsilon$ un nombre réel strictement positif et $y \in X(K)$ un point distinct de $x$ tel que

$$
\log \left(d_{v}(x, y)\right)<-\left(n_{v} / \gamma+\varepsilon\right) h_{\mathscr{L}}(y)
$$

Soit $\widetilde{s} \in \mathrm{H}^{0}\left(\widetilde{X}, r m \mathscr{L}_{\gamma}\right) \backslash\{0\}$. D'après le lemme 5.4 , il existe une section non nulle $s \in \mathrm{H}^{0}\left(X, \mathscr{L}^{r m} \otimes I_{x}^{r m \gamma}\right)$ telle que $\widetilde{s}_{\mid \widetilde{X} \backslash E}=\pi^{*}\left(s_{\mid X \backslash\{x\}}\right)$. On peut supposer que $h_{\mathscr{L}}(y)>\left(\frac{n_{v}}{\gamma}+\varepsilon\right)^{-1} a(v) \log 2$; ainsi l'hypothèse du lemme 5.2 est satisfaite et d'après la condition $(A)$ on a

$$
\begin{aligned}
\left\|\widetilde{s}\left(\pi^{-1}(y)\right)\right\|_{v}=\|s(y)\|_{v} & \leq\left(2^{a(v)} \frac{d_{v}(x, y)}{\rho_{x}(\mathscr{L})}\right)^{r m \gamma}\|s\|_{v, \text { sup }} \\
& \leq\left(2^{a(v)} \frac{d_{v}(x, y)}{\rho_{x}(\mathscr{L})}\right)^{r m \gamma}\|\widetilde{s}\|_{v, \text { sup }} .
\end{aligned}
$$

Par ailleurs,

$$
\begin{aligned}
h_{r m \mathscr{L}}(y) & =-\sum_{v \in \Sigma_{L}} \frac{\left[L_{v}: \mathbb{Q}_{v}\right]}{[L: \mathbb{Q}]} \log \|s(y)\|_{v} \\
& =-\sum_{v \in \Sigma_{L}} \frac{\left[L_{v}: \mathbb{Q}_{v}\right]}{[L: \mathbb{Q}]} \log \left\|\widetilde{s}\left(\pi^{-1}(y)\right)\right\|_{v}=h_{r m \mathscr{L}_{\gamma}}\left(\pi^{-1}(y)\right) .
\end{aligned}
$$

On raisonne maintenant comme dans la preuve du théorème 5.3 en appliquant le corollaire 4.2 à $\mathrm{H}^{0}\left(\widetilde{X}, r m \mathscr{L}_{\gamma}\right)$ au lieu de $F_{r}$. La constante $c^{\prime}(\mathscr{L}, x)$ qui apparaît à la fin de la démonstration du théorème 5.3 est alors remplacée par $\frac{1}{m} \lambda_{\max }\left(\overline{r m \mathscr{L}_{\gamma}}\right)$. D'après la proposition 2.10 , on a

$$
\lim _{r \rightarrow+\infty} \frac{1}{r} \lambda_{\max }\left(\overline{r m \mathscr{L}_{\gamma}}\right)=-\mathrm{e}\left(\overline{m \mathscr{L}_{\gamma}}\right)
$$

En utilisant à nouveau la condition $(A)$, on obtient

$$
\begin{aligned}
\mathrm{e}\left(\overline{m \mathscr{L}_{\gamma}}\right) & =\inf \left\{h_{m \mathscr{L}_{\gamma}}(z) \mid z \in \widetilde{X}(\overline{\mathbb{Q}})\right\} \\
& =m \min \left(\inf _{\substack{a \in X(\overline{\mathbb{Q}}) \\
a \neq x}} h_{\mathscr{L}}(a), h_{\mathscr{L}}(x)+\gamma \inf \left\{h_{\mathscr{O}(-E)}(z) \mid z \in E(\overline{\mathbb{Q}})\right\}\right) \\
& \geq m \mathrm{e}(\overline{\mathscr{L}})+m \gamma \min \left\{0, \inf _{z \in E(\overline{\mathbb{Q}})} h_{\mathscr{O}(-E)}(z)\right\},
\end{aligned}
$$

ce qui achève la preuve. 
Voyons maintenant comment le corollaire 5.5 implique le corollaire 1.5. Ainsi que nous l'avons rappelé dans la partie 3 , si $\mathscr{L}$ est ample alors $\epsilon_{x}(\mathscr{L})>0$. Considérons un nombre réel strictement positif $\gamma<\epsilon_{x}(\mathscr{L})$; alors pour $r$ suffisamment grand, $\mathscr{B}\left(r \mathscr{L}_{\gamma}\right)=\emptyset$. Soit $\varepsilon>0$ et soit $y \in X(K)$ un point distinct de $x$. Si $\gamma$ est suffisamment proche de $\epsilon_{x}(\mathscr{L})$ et si $h_{\mathscr{L}}(y) \geq 0$, on a l'implication

$$
\begin{aligned}
& \log \left(d_{v}(x, y)\right)<-\left(\frac{[L: \mathbb{Q}]}{\epsilon_{x}(\mathscr{L})\left[L_{v}: \mathbb{Q}_{v}\right]}+\varepsilon\right) h_{\mathscr{L}}(y) \\
& \Longrightarrow \log \left(d_{v}(x, y)\right)<-\left(\frac{[L: \mathbb{Q}]}{\gamma\left[L_{v}: \mathbb{Q}_{v}\right]}+\frac{\varepsilon}{2}\right) h_{\mathscr{L}}(y) .
\end{aligned}
$$

On obtient alors le corollaire 1.5 en posant

$$
b(\mathscr{L}, x)=\varepsilon\left(\frac{[L: \mathbb{Q}]}{\gamma\left[L_{v}: \mathbb{Q}_{v}\right]}\right)^{-1} a(v) \log 2+2 \max \left\{0, c_{1}(\mathscr{L}, x)+c_{2}(\mathscr{L}, x, \gamma, r)\right\},
$$

où $c_{1}(\mathscr{L}, x)$ et $c_{2}(\mathscr{L}, x, \gamma, r)$ sont les constantes du corollaire 5.5. De plus, si l'hypothèse du théorème 5.8 est satisfaite, on peut remplacer la constante $c_{2}(\mathscr{L}, x, \gamma, r)$ par la constante $c_{3}(\mathscr{L}, x, \gamma)$. La propriété de Northcott implique qu'il n'existe qu'un nombre fini de points $y \in X(K)$ distincts de $x$ tels que

$$
\log \left(d_{v}(x, y)\right)<-\left(\frac{[L: \mathbb{Q}]}{\epsilon_{x}(\mathscr{L})\left[L_{v}: \mathbb{Q}_{v}\right]}+\varepsilon\right) h_{\mathscr{L}}(y)
$$

Dans le cas où le point $x$ n'est plus supposé régulier, les mêmes méthodes permettent de majorer la hauteur des points vérifiant (8), mais dans ce cas la constante qui intervient n'est plus explicite. L'inconvénient est que le lemme 5.2 ne s'applique pas et l'hypothèse du corollaire 4.2 n'est plus garantie. Nous pouvons cependant utiliser le corollaire 4.4 ; expliquons maintenant comment procéder. Soient $\gamma$ un nombre rationnel et $r$ un entier non nul tel que $r \gamma \in \mathbb{N}, \varepsilon$ un nombre réel strictement positif et $y \in(X \backslash \mathscr{B})(K)$ ( $\mathscr{B}$ désigne encore l'image par $\pi$ du lieu de base de $r \mathscr{L}_{\gamma}$ ) tel que

$$
\log \left(d_{v}(x, y)\right)<-\left(\frac{[L: \mathbb{Q}]}{\gamma\left[L_{v}: \mathbb{Q}_{v}\right]}+\varepsilon\right) h_{\mathscr{L}}(y) .
$$

On note $V=\mathrm{H}^{0}(X, r \mathscr{L})$ et $F_{r}$ le sous-espace de $V$ des sections s'annulant à l'ordre $r \gamma$ en $x$. On considère la filtration de $V$ donnée par le drapeau

$$
V \supset F_{r} \supset\{0\}
$$

et les nombres réels $c_{0}=0<c_{1}=\frac{[L: \mathbb{Q}]}{\left[L_{v}: \mathbb{Q}_{v}\right]}+\gamma \varepsilon / 2$. Il existe des générateurs $\left(u_{1}, \ldots, u_{t}\right)$ de l'idéal maximal défini par $x$ tels que si $d_{v}(x, z)$ est suffisamment petite, alors

$$
\max \left(\left|u_{1}(z)\right|_{v}, \ldots,\left|u_{t}(z)\right|_{v}\right) \leq 2^{a(v)} d_{v}(x, z)
$$

(on utilise ici les mêmes arguments qu'au début de la preuve du lemme 5.2 ainsi que le lemme 5.1). On en déduit que si la hauteur de $y$ est suffisamment grande, $\left|u_{i}(y)\right|_{v} \leq 2^{a(v)} d_{v}(x, y)$ pour tout $1 \leq i \leq t$. En considérant un recouvrement de $\widetilde{X}$ par des ouverts affines et l'égalité

$$
r \mathscr{L}_{\gamma}=\left(\pi^{-1} \mathscr{L}\right)^{\otimes r} \otimes_{\pi^{-1} \mathscr{O}_{X}}\left(\pi^{-1} I_{x}\right)^{\otimes r \gamma} \otimes_{\pi^{-1} \mathscr{O}_{X}} \mathscr{O}_{\widetilde{X}},
$$


(voir la preuve du lemme 5.4), on peut supposer que $y$ n'appartient pas à $\mathscr{B}\left(F_{r}\right)$. Soit $U$ un ouvert de $X$ contenant $x$ et $s_{0} \in \mathrm{H}^{0}(X, r \mathscr{L})$ une section ne s'annulant pas sur $U$. On choisit une base $\left(s_{1}, \ldots, s_{\operatorname{dim} F}\right)$ de $F_{r}$ avec

$$
s_{i}=s_{0} \cdot f_{i} \sum_{\substack{\alpha_{i 1}+\cdots+\alpha_{i t}=m r \gamma \\ \alpha=\left(\alpha_{i 1}, \ldots, \alpha_{i t}\right)}} a_{\alpha} u_{1}^{\alpha_{i 1}} \cdots u_{r}^{\alpha_{i t}},
$$

où $f_{i} \in \mathscr{O}_{X}(U)$ et

$$
\left\|s_{0} \cdot f_{i}\right\|_{v, \sup } \sum_{\alpha}\left|a_{\alpha}\right|_{v} \leq 1 .
$$

Pour tout $1 \leq i \leq \operatorname{dim} F$, on a ainsi

$$
\left\|s_{i}(y)\right\|_{v} \leq \max _{1 \leq j \leq t}\left\{\left|u_{j}\right|_{v}\right\}^{r \gamma} \leq\left(2^{a(v)} d_{v}(x, y)\right)^{r \gamma} .
$$

En raisonnant comme dans la preuve du corollaire 5.5 et en utilisant le corollaire 4.4, on majore ainsi la hauteur des points satisfaisant le système (8). Cette majoration fait intervenir la constante du corollaire 4.4 (pour le cas particulier d'une place) qui dépend malheureusement de la base $\left(s_{1}, \ldots, s_{\operatorname{dim} F}\right)$ choisie pour obtenir (9). Ces méthodes redonnent ainsi une généralisation du théorème de Liouville même dans le cas où le point $x$ n'est pas régulier.

Le corollaire 5.5 entraîne la minoration $\alpha_{x}(\mathscr{L}) \geq \frac{1}{[L: K]} \epsilon_{x}(\mathscr{L})$ du corollaire 3.4 de $[14]$ (le facteur $\left[L_{v}: \mathbb{Q}_{v}\right]$ qui apparaît dans le corollaire 5.5 provient d'une différence de normalisation) et fournit une majoration pour la hauteur des points qui ne dépend pas d'un choix de métriques sur $E$. Remarquons que dans [13], les auteurs démontrent la minoration plus forte $\alpha_{x}(\mathscr{L}) \geq \frac{1}{2} \epsilon_{x}(\mathscr{L})$, qui généralise le théorème de Roth. Mais même dans le cas de la droite projective $X=\mathbb{P}_{K}^{1}$, aucune version effective n'existe à l'heure actuelle pour le théorème de Roth.

\subsection{Le cas de $\mathbb{P}^{n}$}

Dans ce paragraphe, nous considérons le cas où $X=\mathbb{P}_{K}^{n}$ et $\mathscr{L}=\mathscr{O}(1)$. Dans le cas où $n \geq 2$, l'exposant d'approximation du corollaire 5.5 peut être amélioré, tout en conservant une majoration effective de la hauteur des solutions. Nous proposons une généralisation de la version effective du théorème de Liouville, analogue à (1). Soit $x=\left(x_{0}: \ldots: x_{n}\right)$ un point de $\mathbb{P}^{n}(\bar{K}) \backslash \mathbb{P}^{n}(K)$ et soit $v$ une place de $\bar{K}$. Par abus de notation, on note encore $v$ la restriction de cette place à des sous-corps de $\bar{K}$. Sans perte de généralité, on peut supposer que $x_{0} \neq 0$ et on note $x=\left(1: \widetilde{x}_{1}: \cdots: \widetilde{x}_{n}\right)$.

Théorème 5.9. On note

$$
\theta=\min \left\{\frac{\left[K\left(\widetilde{x}_{i}\right): \mathbb{Q}\right]}{\left[K\left(\widetilde{x}_{i}\right)_{v}: \mathbb{Q}_{v}\right]} \mid \widetilde{x}_{i} \notin K\right\} .
$$

Pour tout point $y=\left(y_{0}: \ldots: y_{n}\right) \in \mathbb{P}^{n}(K)$ tel que $y_{0} \neq 0$, on a

$$
\log \left(d_{v}(x, y)\right)>-\theta\left(h_{2}(y)+h_{2}(x)\right) .
$$


Démonstration : Soit $1 \leq i \leq n$ tel que $\theta=\left[K\left(\widetilde{x}_{i}\right): \mathbb{Q}\right] /\left[K\left(\widetilde{x}_{i}\right)_{v}: \mathbb{Q}_{v}\right]$ et $\widetilde{x}_{i} \notin K$. On note $L=K\left(\widetilde{x}_{i}\right)$. Considérons la section

$$
s=\widetilde{x}_{i} T_{0}-T_{i} \in \mathrm{H}^{0}\left(\mathbb{P}_{L}^{n}, \mathscr{O}(1)\right) \backslash\{0\} .
$$

Soit $y \in \mathbb{P}^{n}(K)$ tel que $y_{0} \neq 0$. Alors $s(y) \neq 0$ car $\widetilde{x}_{i} \notin K$ et $y \in \mathbb{P}^{n}(K)$. On considère la métrique sur $\mathscr{O}(1)$ telle que $h_{\overline{\mathscr{O}(1)}}(y)=h_{2}(y)$ définie dans l'exemple 2.6. Supposons que la place $v$ est finie. Alors

$$
\begin{aligned}
\|s(y)\|_{v}=\frac{\left|y_{i}-y_{0} \widetilde{x}_{i}\right|_{v}}{\max _{0 \leq j \leq n}\left|y_{j}\right|_{v}} & \leq \frac{\max _{0 \leq k<l \leq n}\left|y_{l}-y_{k} \widetilde{x}_{l}\right|_{v}}{\max _{0 \leq j \leq n}\left|y_{j}\right|_{v}} \\
& =d_{v}(x, y) \cdot \max \left\{1,\left|\widetilde{x}_{1}\right|_{v}, \ldots,\left|\widetilde{x}_{n}\right|_{v}\right\} .
\end{aligned}
$$

Dans le cas où $v$ est archimédienne, on a de la même façon

$$
\begin{aligned}
\|s(y)\|_{v}=\frac{\left|y_{i}-y_{0} \widetilde{x}_{i}\right|_{v}}{\left|\left(y_{0}, \ldots, y_{n}\right)\right|_{2, v}} & \leq \frac{\left|\left(y_{l}-y_{k} \widetilde{x}_{l}\right)_{0 \leq k<l \leq n}\right|_{2, v}}{\left|\left(y_{0}, \ldots, y_{n}\right)\right|_{2, v}} \\
& =d_{v}(x, y) \cdot\left|\left(1, \widetilde{x}_{1}, \ldots, \widetilde{x}_{n}\right)\right|_{2, v} .
\end{aligned}
$$

Pour toute place $w \in \Sigma_{L}$, on a par ailleurs

$$
\|s(y)\|_{w} \leq\|s\|_{w, \sup } \leq\left|\left(1, \widetilde{x}_{i}\right)\right|_{2, w} \leq\left|\left(1, \widetilde{x}_{1}, \ldots, \widetilde{x}_{n}\right)\right|_{2, w} .
$$

On en déduit que $-h_{2}(y) \leq h_{2}(x)+\frac{1}{\theta} \log d_{v}(x, y)$, ce qui achève la preuve.

Dans le cas où $n=1$, l'exposant $\theta$ est égal à l'exposant $\frac{[L: \mathbb{Q}]}{\left[L_{v}: \mathbb{Q} v\right]}$ du corollaire 5.5 , où $L$ est le corps de définition de $x$. Cependant dès que $n \geq 2$, l'exposant du théorème 5.9 peut être strictement inférieur à $\frac{[L: \mathbb{Q}]}{\left[L_{v}: \mathbb{Q}_{v}\right]}$. Remarquons que dans l'article [13], les auteurs obtiennent un meilleur exposant $\left(\frac{m}{m+1}\right.$, où $m$ est la dimension du plus petit sous-espace linéaire contenant $\left.x \in \mathbb{P}^{n}(\bar{K}) \backslash \mathbb{P}^{n}(K)\right)$. Une fois de plus, l'intérêt du théorème 5.9 est qu'il donne une majoration explicite de la hauteur des solutions.

\section{Références}

[1] Huayi CHEN : Convergence des polygones de Harder-Narasimhan. Mém. Soc. Math. Fr. (N.S.), (120):116, 2010.

[2] Huayi Chen et Catriona Maclean : Distribution of logarithmic spectra of the equilibrium energy. Manuscripta Math., 146(3-4):365-394, 2015.

[3] P. R. Chernoff, R. A. Rasala et W. C. Waterhouse : The StoneWeierstrass theorem for valuable fields. Pacific J. Math., 27:233-240, 1968.

[4] Kwok-Kwong ChOI et Jeffrey D. VAALER : Diophantine approximation in projective space. In Number theory (Ottawa, ON, 1996), volume 19 de CRM Proc. Lecture Notes, pages 55-65. Amer. Math. Soc., Providence, RI, 1999.

[5] Jean-Pierre Demailly : Singular Hermitian metrics on positive line bundles. In Complex algebraic varieties (Bayreuth, 1990), volume 1507 de Lecture Notes in Math., pages 87-104. Springer, Berlin, 1992. 
[6] Gerd Faltings et Gisbert WÜstholz : Diophantine approximations on projective spaces. Invent. Math., 116(1-3):109-138, 1994.

[7] Carlo Gasbarri : Transcendental Liouville inequalities on projective varieties. arXiv :1609.04262.

[8] Éric Gaudron : Pentes des fibrés vectoriels adéliques sur un corps global. Rend. Semin. Mat. Univ. Padova, 119:21-95, 2008.

[9] Marc Hindry et Joseph H. Silverman : Diophantine geometry, volume 201 de Graduate Texts in Mathematics. Springer-Verlag, New York, 2000. An introduction.

[10] Christine Laurent-ThiéBaut : Théorie des fonctions holomorphes de plusieurs variables. Savoirs Actuels. [Current Scholarship]. InterEditions, Paris; Masson, Paris, 1997. Mathématiques. [Mathematics].

[11] Robert LazARsfeld : Positivity in algebraic geometry. I, volume 48 de Ergebnisse der Mathematik und ihrer Grenzgebiete. 3. Folge. A Series of Modern Surveys in Mathematics [Results in Mathematics and Related Areas. 3rd Series. A Series of Modern Surveys in Mathematics]. Springer-Verlag, Berlin, 2004. Classical setting : line bundles and linear series.

[12] Qing LiU : Algebraic geometry and arithmetic curves, volume 6 de Oxford Graduate Texts in Mathematics. Oxford University Press, Oxford, 2002. Translated from the French by Reinie Erné, Oxford Science Publications.

[13] David McKinnon et Mike Roth : Seshadri constants, diophantine approximation, and Roth's theorem for arbitrary varieties. Invent. Math., 200(2):513-583, 2015.

[14] David McKinnon et Mike Roth : An analogue of Liouville's Theorem and an application to cubic surfaces. Eur. J. Math., 2(4):929-959, 2016.

[15] James S. Milne : Étale cohomology, volume 33 de Princeton Mathematical Series. Princeton University Press, Princeton, N.J., 1980.

[16] Atsushi Moriwaki : Arakelov geometry, volume 244 de Translations of Mathematical Monographs. American Mathematical Society, Providence, RI. Translated from the 2008 Japanese original.

[17] Atsushi Moriwaki : Semiample invertible sheaves with semipositive continuous hermitian metrics. Algebra Number Theory, 9(2):503-509, 2015.

[18] Damien Roy et Jeffrey Lin Thunder : Addendum and erratum to : "An absolute Siegel's lemma" [J. Reine Angew. Math. 476 (1996), 1-26; MR1401695 (97h :11075)]. J. Reine Angew. Math., 508:47-51, 1999.

[19] Paul VojTA : Applications of arithmetic algebraic geometry to Diophantine approximations. In Arithmetic algebraic geometry (Trento, 1991), volume 1553 de Lecture Notes in Math., pages 164-208. Springer, Berlin, 1993.

[20] Shouwu Zhang : Positive line bundles on arithmetic varieties. J. Amer. Math. Soc., 8(1):187-221, 1995. 